\title{
Double-Layer Network Model of Bank-Enterprise Counterparty Credit Risk Contagion
}

\author{
Tingqiang Chen $\left(\mathbb{D},{ }^{1,2}\right.$ Qinghao Yang, ${ }^{1,2}$ Yutong Wang, ${ }^{1}$ and Suyang Wang ${ }^{3}$ \\ ${ }^{1}$ School of Economic and Management, Nanjing Tech University, Nanjing 211816, China \\ ${ }^{2}$ Research Center of Big Data Decision and Performance Evaluation, Nanjing Tech University, Nanjing 211816, China \\ ${ }^{3}$ Shanghai Stock Exchange, Shanghai 200120, China
}

Correspondence should be addressed to Tingqiang Chen; tingqiang88888888@163.com

Received 21 June 2020; Revised 16 July 2020; Accepted 22 July 2020; Published 30 October 2020

Academic Editor: Dimitri Volchenkov

Copyright (c) 2020 Tingqiang Chen et al. This is an open access article distributed under the Creative Commons Attribution License, which permits unrestricted use, distribution, and reproduction in any medium, provided the original work is properly cited.

\begin{abstract}
Banks and enterprises constitute a multilayered, multiattribute, multicriteria credit-related super network due to financial transaction behaviors, such as credit, wealth management, savings, and derivatives. Such a network has become an important channel for credit risk cross-contagion. This study constructs a two-layer network model of credit risk contagion between the bank and corporate counterparties from the perspective that banks do not withdraw loans from enterprises by considering the influence of corporate credit defaults on their counterparties under the credit linkage. This study analyzes the mechanism of influencing the evolution of bank-enterprise counterparty credit risk contagion in the two-tier network through theoretical analysis, including the following: the enterprises' coping ability, risk preference, influence, level of interenterprise credit risk contagion and its network heterogeneity in the interenterprise credit association network, the risk prevention and control ability, business correlation degree, interbank credit risk contagion and its network heterogeneity in the interbank credit association network, the level of credit risk contagion between bank-enterprise counterparty credit association networks, and other factors in the case that banks do not withdraw loans from enterprises. In addition, this study performs a calculation experiment to analyze the characteristics of the evolution of counterparty credit risk contagion of bank and corporate counterparties under the double-layer network. The following four major conclusions can be drawn from the results. First, in the interenterprise credit-related network, the threshold of credit risk contagion rate is positively correlated with the marginal increase in risk perception and risk leveling ability of the enterprise. By contrast, such threshold is negatively correlated with the marginal decrease in the initial economic impact, leverage level, and influence of the enterprise. Moreover, the scale of corporate counterparty credit risk contagion is negatively correlated with the enterprise's risk perception level and risk spillover ability but positively correlated with the enterprise's initial economic shock level, the enterprise's leverage level, and influence. Second, in the interbank credit association network, the threshold of the rate of credit risk contagion is negatively correlated with the marginal decrease in the degree of interbank business association but positively correlated with the marginal increase in the bank's risk resistance ability and risk information processing ability. Furthermore, the scale of credit risk contagion of bank counterparties is positively correlated with the degree of interbank business association but negatively correlated with the bank's ability to resist risks and process risk information. Third, if the heterogeneity of the credit-related network of bank-enterprise counterparties is high, then the rate threshold of credit risk contagion is high and the scale of credit risk diffusion is low. Moreover, the scale of credit risk contagion of bank counterparties is positively correlated with the marginal decrease in the degree of corporate and bank counterparties. Finally, the scale of bank counterparty credit risk contagion is a monotonically increasing convex function of the credit risk contagion rate in the enterprise credit association network and among the bank-enterprise networks.
\end{abstract}




\section{Introduction}

Corporate default is a ubiquitous economic phenomenon, which is prominent during the economic recession. An enterprise's default will often lead to dependent default or even bankruptcy of the enterprise related to its business or credit. Thus, a "domino effect" of default risk contagion has formed in the network of enterprises with business or credit relationship, which can lead to systemic risk and local credit crisis in severe cases. Simultaneously, the complexity of the impact of corporate defaults has increased due to the deep participation of banks and other financial entities, which often leads to a wide contagion of corporate default and serious socioeconomic impact. Typical examples include the US subprime mortgage crisis in 2007 and the European debt crisis in 2010.

In the modern economic system, the symbiotic relationship between banks and enterprises is becoming increasingly evident, and bank-enterprise credit provides endless financial support for enterprise development. However, enterprises will face multiple risks in the process of business development due to the interaction between their own internal factors and the external macroeconomic environment. Once corporate risk exposure inevitably leads to credit default of enterprises on banks, the credit risk of an enterprise will be transmitted to the bank through the credit network. Moreover, an infectious effect will be formed in the bank-enterprise credit-related network. At present, academic research on credit risk contagion of banks and enterprises mainly focuses on the following three aspects. First, with the increasing degree of financial liberalization, the loan transactions between banks and enterprises have increased substantially, and the credit risks of loans have continuously infected banks and enterprises [1-8]. The bank-enterprise credit-linked network has the role of a financial accelerator. Some scholars have conducted corresponding research on the bank-enterprise credit association network and its characteristics. For instance, Masi and Gallegati [9] studied the Italian bank-enterprise credit network and found that the credit connection between banks and enterprises is heterogeneous. Asanuma [10] built a credit network through multiple enterprises and a single bank, considering the bank borrowing attitudes and borrowing enterprises by credit network directly and indirectly connected with bank loans. Gatti et al. [11] studied the characteristics of the network economy in the three sectors of upstream and downstream enterprises and banks. They found that the network structure between banks and enterprises and the nonperforming loan ratio involved can lead to bankruptcy of one or more enterprises. The bankruptcy risk would be transmitted to other enterprises or banks through the bank-to-enterprise credit connection network, causing bankruptcy of other enterprises or banks. Second, the interenterprise guarantee risk influenced by the expansion of the credit market and the economic shocks has caused tremendous trauma to the real economy and the banking industry [12-15]. However, the moral hazard or adverse selection in the bank-enterprise credit market is one of the important factors of corporate guarantee risk. Yin et al. [16] used a unique dataset obtained from a major state-owned commercial bank in China and models that explicitly incorporate heterogeneous borrower qualities. They analyzed the risk of default and provision of collateral for bank loans to firms with varied credit qualities and found that low-quality borrowers may be required to provide collateral at the bank's request to mitigate moral hazard problems. On the contrary, high-quality borrowers may provide collateral willingly to signal quality to mitigate adverse selection problem when competing for obtaining access to bank loans. Liang et al. [17] employed the stochastic frontier approach on 14 banks over the 2001-2010 period to examine how small- and medium-sized enterprises' (SMEs') lending and credit guarantee affect Korean banks' efficiency. They found that when increasing loans to SMEs, the cost efficiency of banks decreases due to information asymmetry. However, increasing the proportion of credit guarantee can improve their cost efficiency. This finding proves that the credit guarantee system of South Korea can share the credit risk of SMEs to help improve bank efficiency. In addition, Gropp et al. [18] conducted a natural experiment using matched bank/firm data to identify the effects of bank guarantees on allocative efficiency. The study found that unproductive firms with guarantees in place receive larger loans, invest more, and maintain higher rates of sales and wage growth compared with those without. Finally, the market impact caused by the macroenvironment poses a threat to a single enterprise or multiple enterprises. The negative impact faced by a single enterprise may affect the entire bank-enterprise market and produce a contagion effect [11, 19-24]. Li and Sui [25] studied the credit risk contagion of banks and upstream and downstream enterprises' counterparties based on the perspective of credit. They concluded that the quality of credit assets in the financial system has declined due to market shocks caused by the macro environment. Moreover, the probability of credit asset downgrade or even default between banks and enterprises has increased, resulting in the loss of banks with credit relationship. Mohammad et al. [26] believed that the individual risk, credit risk, and liquidity risk caused by risk shock will be transmitted to the network counterparties of banks and enterprises through stratified and partial transmissions, which will further cause the extensive spread of bank-enterprise counterparty credit risks. Asanuma [10] believed that although each borrower directly establishes contact with the lender through its credit contract, each borrower is indirectly connected with all other borrowers in the credit network. The study found that the bank's lending attitude can be used as a financial accelerator, which greatly affects the dynamics of the economic system through the credit network. The same level of external shock will produce completely different results according to the lending attitude of different banks.

With the rapid development of complex networks, such networks provide an effective tool for analyzing and solving the interaction of financial systems [27-31]. In the financial system, the credit association between banks and enterprises constitutes a relatively complex credit association network, which has become an important channel for credit risk and credit crisis contagion [12, 32-35]. Initially, scholars mainly 
studied the credit risk contagion of counterparties on the single-layer network of credit association. For instance, Bargigli and Gallegati [36] took credit connections as the main source of systemic risk under the complex network and described the community of the bank-enterprise credit network in Japan. They found a subset of tightly connected nodes in the credit network (these subsets may conduct any impact affecting the single-tier credit network). Chen et al. [13] constructed a network model of credit risk contagion in the interbank lending market based on time series. Under the single-layer network, they studied how the contagion effects of credit risk accumulate and spread in the interbank market network, and the evolution characteristics of credit risk contagion caused by the initial default of debt banks in the interbank market. Montagna and Kok [37] pointed out that the systemic risk between banks and enterprises through the associated network which is usually greater than the sum of the credit risks considered separately. In the single-layer credit network, the contagion effect of counterparty credit risks of banks and enterprises shows a significant nonlinear feature. Jiang and Fan [38] introduced the transmission dynamics theory in complex networks into the coupling relationship between credit risk contagion and emotional contagion based on the structure of linked credit risk network. They analyzed the evolution process of individual risk contagion and emotional contagion in networks and further investigated the impact of network structure, individual risk attitude, individual risk tolerance, and financial market regulation on credit risk contagion and emotional contagion. Li and Zhou [39] gave the associated credit risk model with the imperfect immunization scenario in the enterprise cluster based on assets related to the framework of the smallworld network. They applied mean field theory and principle of epidemiology to reveal the relationship between associated credit risk contagion probability with imperfect immunization and asset related to the enterprise community network. Chen et al. [8] considered the heterogeneity of counterparties in the credit risk transfer (CRT) market and preferential connection among counterparties to build the CRT market counterparty credit risk contagion of susceptible-infected-recovered-susceptible (SIRS) model. Moreover, they theoretically analyzed the influence mechanism and evolution characteristics of counterparty behavior preference, counterparty credit risk contagion adaptability, regulatory rescue strategy, and other factors on counterparty credit risk contagion in the CRT market under a single-layer network. Based on complex network theory, Wang et al. [24] constructed a counterparty credit risk contagion model by considering investor behavior and information disclosure strategies and analyzed the network topology characteristics and evolution rules of counterparty credit risk contagion under a single-tier network.

However, bank and enterprise counterparties formed a multilayered, multiattribute, multicriteria credit-linked super network due to financial transaction behaviors, such as credit, wealth management, savings, and derivatives. Fully analyzing and depicting the complex mechanism of credit risk contagion among bank-enterprise counterparties from the perspective of a single-layer network are difficult. With the continuous deepening of basic research of complex networks and theoretical breakthroughs, the multilayer network analysis method makes the research results of counterparty infection highly realistic to the actual market. In a credit association network, multilayer network analysis can describe complex credit associations among different types of counterparties in the same layer network and also can describe the interaction among counterparties in different network layers. Moreover, the contagion effect caused by the interaction among different network layers is often greater than that in a single-layer network [3, 23, 35, 40-42]. Wang et al. [43] from the perspective of macroprudential stress testing constructed a risk contagion model of bankreal estate loan network under the double-layer network. Anand et al. [44] studied the role of macroeconomic fluctuations, asset market liquidity and network structure on risk contagion, and loss of the financial system in a multilayer network, which was composed of three different but interconnected sets of agents-domestic banks, overseas banks, and firms. Granell et al. [45] analyzed the interaction in the transmission process of infectious diseases based on the two-layer network. They found that the evolution process of the infectious disease threshold can be explored according to the relationship between the topological structure of the two-layer network and the ideology. Iori et al. [46] measured the effect of individual infectors on systemic risk in the three-layer network of long- and shortterm loans and holding common assets based on the multilayer network. They concluded that the multilayer network measurement of systemic risk was greater than that of the aggregate risk of each layer of the network, and the systemic risk was related to the financial multilayer network topology. Hüser et al. [47] used granular data on the security cross-holdings among the largest euro area banking groups and constructed a multilayered network model. Each layer represents bail-inable securities of a specific seniority layer of the creditor hierarchy, which can be a useful tool for resolution authorities to inform the policy discussion on the composition and level of loss-absorbing capacity of banks and the direct contagion risk following a bail-in. Ding et al. [6] constructed a bidirectional bank network composed of credit relationship and information interaction through the balance sheets of banks, modeled the structure of systemic risk, and studied the dynamic mechanism of the spread of default risk and liquidity contagion and its influencing factors. Silva et al. [33] built a feedback mechanism model for bank-enterprise based on a multilayer network. They believed that the feedback between enterprises and banks in the financial system can easily magnify systemic risks; that is, the shock from the enterprise will be transmitted to the bank through the financial network. This case in turn will not only amplify the initial impact of the enterprise but also bring deterioration in the financial situation. From the perspective of stock price fluctuation, Dong et al. [48] constructed a single-layer network SCIR investor risk contagion model considering the heterogeneity of investors based on the single-layer network SCIR investor risk contagion model. They constructed a two-layer coupling network investor risk contagion model and compared the evolution characteristics 
and rules of investor risk contagion under different connection modes and mechanism probabilities. Chen et al. [8] considered the potential default status and counterparty heterogeneity of counterparties in the CRT market. They constructed a counterparty credit risk contagion model under a double-layer coupling network based on the singlelayer network model and analyzed the evolution behavior characteristics of counterparty credit risk contagion under the two-layer coupling network.

In summary, under the credit correlation network, existing studies have constructed and analyzed the model of banks and enterprise credit risk transmission and its evolution with the help of complex networks. However, research for intra- and internetwork infections under a double-layer network that considered the heterogeneity of banks and enterprises and their risk behavior attributes and integrated the infectious disease model to study the bank and enterprise counterparty credit risk transmission is limited. Therefore, this study considers the impact of corporate credit defaults on their counterparties under the credit linkage based on the perspective that banks do not withdraw loans from enterprises. Then, this study constructs a two-layer network model of credit risk contagion between bank and enterprise counterparties and analyzes in depth the following: response ability, risk preference, influence, level of interenterprise credit risk contagion and its network heterogeneity in the interenterprise credit association network, the risk prevention and control ability, business correlation degree, interbank credit risk contagion level and its network heterogeneity in interbank credit association network, and factors, such as the level of credit risk contagion between bank-enterprise counterparty credit-related networks. Furthermore, this study discusses the influence mechanism and evolution characteristics of these factors on the credit risk contagion of bank-enterprise counterparty under the twolayer network.

The structure of this paper is as follows: Section 2 analyzes the credit risk contagion mechanism of bank-enterprise counterparties under the double-layer network. Section 3 constructs the credit risk contagion model of bankenterprise counterparties under the double-layer network. Section 4 simulates and analyzes the evolution characteristics of credit risk contagion of bank-enterprise counterparties under the double-layer network. Section 5 summarizes and concludes the study.

\section{Credit Risk Contagion Behavior Mechanism of Bank-Enterprise Counterparties under a Double- Layer Network}

In the modern economic system, the business connection based on credit connection between banks and nonbanking companies is becoming increasingly close. Moreover, a significant symbiotic relationship is observed between banks and enterprises. However, with the deep development of the business relationship between banks and enterprises, the subject transactions based on credit are also becoming frequent. In addition, the credit relationship between banks and enterprises based on credit relationships is becoming increasingly complex, which has become an important channel for credit risk contagion. In the enterprise system, various types of enterprises of various sizes have formed a close credit relationship through single or multiple methods, such as cross-shareholding, supply chain relationships, related party transactions, credit guarantees, capital dismantling, and asset-liability relationship. This relationship constitutes an enterprise counterparty credit-related network. In the enterprise credit association network, the financial relationship between companies is inevitable due to these complex and intertwined credit-related relationships. When some companies have fluctuations in credit ratings or increased credit risk or defaults, direct or indirect creditrelated companies will inevitably be affected, forming financially consistent risk gains and losses. In this case, credit risk may be contagious in the enterprise's credit-related network, which may lead to systemic risk or even credit crisis in serious cases.

The interbank lending, joint shareholding, asset-liability relationship, and other complex relationships among the banking departments constitute the bank's credit association network, which forms an important channel for interbank counterparty risk contagion. In the bank credit association network, credit activities, such as credit business, lending business, wealth management product trading business, and bond underwriting business, lead to the formation and dependence of credit risk. A bank default will affect mutual contagion through the bank credit association network, resulting in the linkage effect of interbank assets and liabilities and causing systemic risks. The interbank credit association network plays a crucial role in the interflow of liquidity within the banking system and the redistribution of credit resources. However, the interbank credit association network provides a channel for the spread and diffusion of credit risk.

With the development of the economy, the credit relationship between banks and enterprises is becoming increasingly close. Credit is an important part of the credit activities between banks and enterprises. Nevertheless, credit has become an important channel for the formation of credit dependence between bank and enterprise counterparties. In an uncertain environment, decision-making behaviors and operating benefits of enterprises are easily affected by macro and micro uncertain economic, social, and policy factors. The formation of corporate credit default is hidden, sudden, and contagious, and enterprise defaults can infect the interbank counterparties through the credit relationship between banks and enterprises, resulting in bank credit losses. In addition, the impact of this loss will be transmitted to neighboring banks through the interbank credit-related network, triggering the mutual infection and transmission of bank-enterprise credit risk in such a network. When the bank losses are small, the credit risk transmission is within the controlled range, and the contagion impact is below the critical level, then the bank will not withdraw loans to enterprises. However, if the contagion shock is higher than the critical level of bank risk tolerance, then banks will withdraw 
loans from enterprises, causing additional serious credit risk contagion.

According to the above analysis, some enterprises have experienced credit defaults or increased credit risk levels in the enterprise network $A$ may be due to financial crisis, poor management, and internal and external factors, such as the fluctuation in the macromarket environment influence. Thus, this case leads to a change from healthy companies to default ones and became the source of credit risk in corporate networks $A$. In the enterprise network $A$, the enterprise credit risk is continuously transmitted to the enterprises with a direct or indirect connection through the interenterprise credit-related network. Considering the credit risk level of enterprises and their ability to resist credit risk, the possible credit risk state of an enterprise after participating in credit activities in the actual operation process is divided into three states: healthy state enterprise $S_{A}$, credit default state enterprise $I_{A}$, and bankruptcy state enterprise $R_{A}$. Healthy state enterprises $S_{A}$ refer to credit risk with a low level of risk exposure but are susceptible to the influence of companies with a high level of credit risk associated with them, resulting in credit default risk. Credit default state enterprises $I_{A}$ refer to the influence of internal and external factors, where credit default state enterprises spread to other enterprise nodes at a certain probability. Then, bankrupt state enterprises $R_{A}$ refer to a large scale of defaults affected by internal and external counterparties, causing serious losses and bankruptcy that cannot be restored. However, the bankrupt enterprises can be restored to healthy enterprises through restructuring and introducing new strategic investors.

In the banking network $B$, the bank balance sheet association, jointly owned assets, interbank lending business relationship, and other correlations are all different sizes of bank interconnected link. At the initial moment, some banks may be affected by the decline of asset quality and credit losses, which lead to credit default and become the source of credit risk contagion in network $B$. The banking network $B$ has three types of banks: healthy banks $S_{B}$, which have credit risks with low-risk exposure, but are vulnerable to credit default risks caused by banks with high associated credit risks; default banks $I_{B}$, which are affected by internal and external factors, have a credit default and spread it to other bank nodes with a certain probability; bankruptcy banks $R_{B}$ which suffer a large area of default affected by internal and external counterparties, causing serious losses and facing bankruptcy that cannot be restored. However, bankrupt banks can be restored to healthy ones by restructuring, injecting capital, and bringing in new investors.

In summary, this study considers the credit association between the corporate network and the bank network based on the complex network and SIR infectious disease theory. This study also builds a credit risk infection model SIRS SIRS that includes the interaction between the corporate network and the bank network. When a corporate counterparty defaults due to poor management or the impact of macroeconomic fluctuations, the credit risk gradually forms and spreads in the corporate network and to some banks with poor-risk resistance through the credit association between banks and enterprises. These banks infected with credit risk pass such risk to their neighbors through interbank business association activities, resulting in further transmission of credit risk in the bank network and increasing the impact on the bank network. The part of the bank that is infected with credit risk then passes the credit risk to neighboring banks through interbank business-related activities, causing further contagion of credit risk in the banking network and increasing the impact on the banking network. Among them, Figure 1 shows the credit risk transmission path under the interaction of the corporate and bank networks.

\section{SIRS Model of Credit Risk Contagion of Bank- Enterprise Counterparty}

3.1. Basic Assumptions. This study set the number of nodes in the enterprise and bank networks to $M$ and $N$, respectively, to build an evolutionary model of counterparty credit risk contagion based on enterprise $A$ and bank $B$ networks. According to the previous analysis, the contagion mechanism of credit risk in the double-layer credit association network between banks and enterprises can be abstracted as shown in Figure 2.

Given that the scale of the counterparties of the enterprise's credit-related network remains unchanged, the sum of the nodes of different types of enterprises at any time is equal to the total number of the enterprise's counterparties $M$, that is, to satisfy the relationship: $S_{A}(t)+I_{A}(t)+R_{A}(t)=M$. Similarly, supposing that the scale of counterparties of the bank's network remains unchanged, the sum of the nodes of different types of bank nodes at any time is equal to the total amount of bank counterparties $N$, that is, to satisfy the relationship: $S_{B}(t)+I_{B}(t)+R_{B}(t)=N$. Considering the degree $k_{A}$ in the enterprise credit association network and the degree $k_{B}$ in the bank network, which are abstracted from the internal business association relationship of the network, the degree distribution of the enterprise credit association network and the bank network is $P_{A}\left(k_{A}\right)$ and $P_{B}\left(k_{B}\right)$, respectively. Based on the above analysis, this study makes the following assumptions:

Hypothesis 1. At the initial moment, some companies experienced increased credit risk or credit defaults due to factors, such as financial crisis, poor management, macropolicy, and market environment fluctuations. Such companies have become the source of credit risk in the corporate credit-related network $A$ and pass the credit risk to the credit-related enterprises with the probability of $\lambda_{A}\left(0<\lambda_{A}<1\right)$ to make it into a default state enterprise. The healthy company $S_{A}$ affected by internal and external factors is partly transformed into a default state enterprise with a probability of $\lambda_{A}$ and partly unable to restore due to serious losses, facing bankruptcy, and transformed into the bankruptcy state enterprise $R_{A}$ with a probability of $u_{A}$. The company $I_{A}$ in the default state is affected by internal and external counterparties and will likely break a large-scale breach of contract and cause serious losses. This company is 


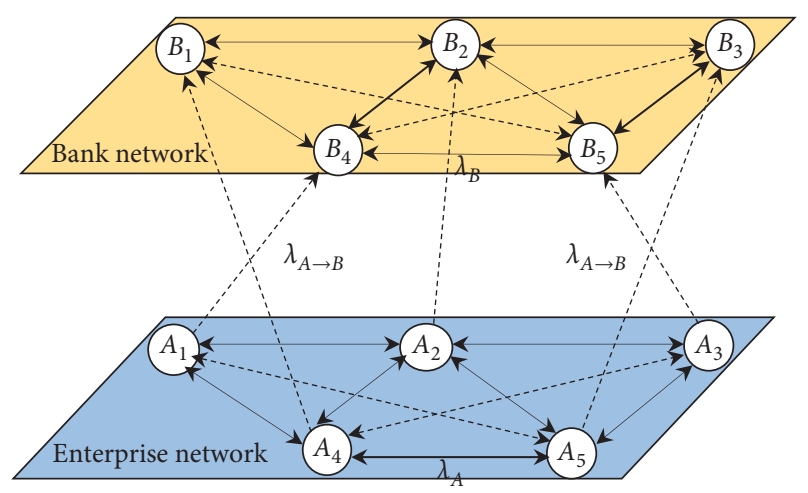

Figure 1: Credit risk transmission path of bank-enterprise counterparties without bank withdrawal loan.

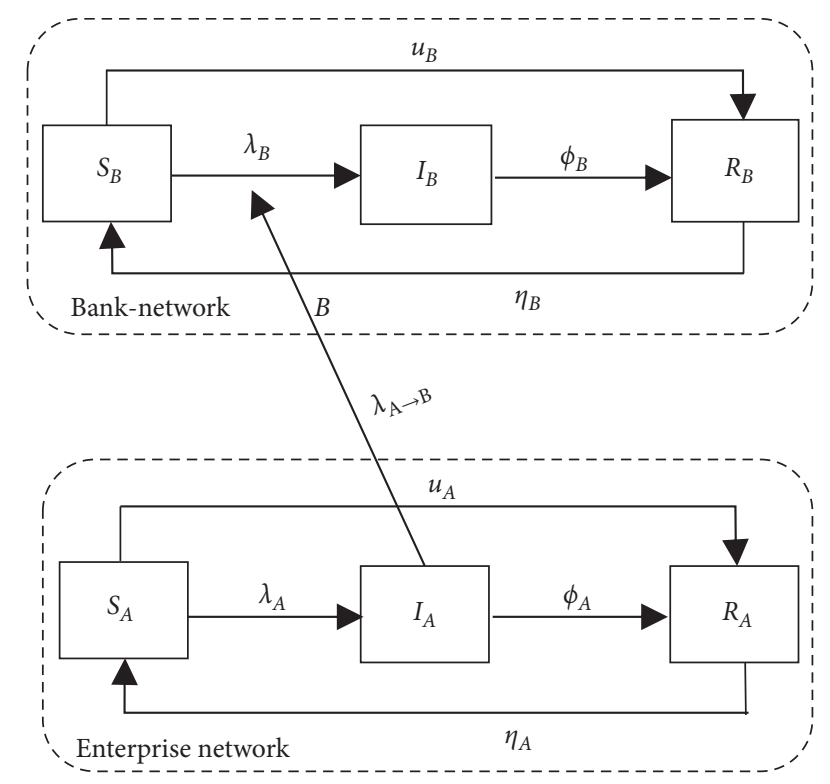

FIGURE 2: Credit risk transmission mechanism of bank-enterprise counterparties without bank withdrawal loan.

transformed into the company $R_{A}$ in bankruptcy with a probability of $\phi_{A}$. The bankruptcy company $R_{A}$ resists the counterparty credit default risk through restructuring and introducing new strategic investors. The bankruptcy company $R_{A}$ will also be transformed into a healthy company $S_{A}$ with a probability of $\eta_{A}$.

Hypothesis 2. At the initial moment, some bank nodes will transfer the credit default risk to the credit-related bank with a probability of $\lambda_{B}\left(0<\lambda_{B}<1\right)$ due to the impact of the decline in the quality of the underlying assets and the impact of credit losses to transform them into default banks. In addition, under the influence of internal and external factors, the healthy state bank $S_{B}$ will also be unable to restore due to severe losses caused by large-scale defaults and will face bankruptcy and change into bankruptcy state bank $R_{B}$ with a probability of $u_{B}$. Under the influence of internal and external counterparties, the default state bank $I_{B}$ produces widespread defaults, causes serious loss, and becomes the bankrupt state bank $R_{B}$ with a probability of $\phi_{B}$. The bank in bankruptcy status $R_{B}$ resists the counterparty credit default risk through reorganization, capital injection, and introduction of new investors and will be transformed into a healthy bank $S_{B}$ with a probability of $\eta_{B}$.

Hypothesis 3. Based on credit association, the default state node $I_{A}$ of the enterprise credit association network has a credit associated with the bank network health state node $S_{B}$. Moreover, the default state node $I_{A}$ of the enterprise credit association network will infect the credit risk to the healthy status node $S_{B}$ of the bank network with a probability of $\lambda_{A \longrightarrow B}\left(0<\lambda_{A \longrightarrow B}<1\right)$ and accelerate or cause its default.

\subsection{Infection Probability}

3.2.1. Probability of Credit Risk Contagion of Corporate Network Counterparty. In an enterprise's credit-related network, companies of different sizes form interrelated relationships in different ways, such as transaction-related, guarantee-related, asset-liability-related, and cross-shareholding. When the enterprise is mismanaged or affected by macroeconomic fluctuations and leads to credit default, these associations provide a channel for credit risk contagion. In enterprise credit association $A$, when corporate counterparties have a credit default due to its own financial crisis, poor management, macroeconomic fluctuations, and so on, the probability of credit risk contagion is influenced by the following five factors:

(1) The initial economic shock $v(0<v<1)$ of the business counterparty. If the initial economic impact on the counterparty of an enterprise is high, then the probability that the counterparty will be converted into a default state is also high [23, 35, 45].

(2) Level $\alpha(0<\alpha<1)$ of corporate counterparty risk perception. The corporate counterparty risk perception level is the perception and recognition of risk by corporate counterparties based on their own knowledge and experience, which is one of the important influencing factors for corporate counterparty decisions $[8,10,12]$. A counterparty with a high level of credit risk awareness, through its own financial resources and analysis of corporate default risk, adopts a strategy to resist credit risk. Therefore, if the level of corporate counterparty risk awareness is high, then the chance of adopting a strategy to resist credit risk is high, and the degree of credit risk contagion is low.

(3) The risk-overflow ability $\tau(0<\tau<1)$ of business counterparties. The ability of counterparty credit risk leveling and overflow reflects the risk hedging level of the counterparty in the process of production and operation. The process is affected by its own financial crisis, mismanagement, macroeconomic environment fluctuations, and in the face of neighbor node 
default $[2,24,49]$. If the counterparty's risk-overflow ability is strong, then the probability of resisting credit defaults is great and that of corporate defaults is small.

(4) The leverage level $\sigma(0<\sigma<1)$ of business counterparties. The leverage level of corporate counterparties is an important factor that exacerbates corporate counterparty default $[9,50]$. If the leverage of corporate counterparties is high, then the exposure to credit risk contagion is great.

(5) The influence $\theta_{i}$ of the counterparty of an enterprise is related to the topology of the enterprise's credit association network $[34,35,51]$. In this study, the influence of the counterparty is measured by the marginal weight $\gamma$ and the degree $k$ of the corporate counterparty, which can be expressed as the weighted average of the marginal weights between the corporate counterparty and $k$ connected counterparties: $\theta_{i}=\sum \gamma_{i} / k_{i}$. Among them, $\sum \gamma_{i}$ represents the sum of the marginal weights associated with the counterparty $i$, and the marginal weights refer to the number of business associations of business counterparties in the recent period. $k_{i}$ represents the degree of counterparty $i$ and refers to the number of sides connected to the counterparty $i$.

Therefore, based on the above analysis, drawing on the credit risk conversion model of Luo et al. [52], the credit risk probability model of corporate counterparties is defined as follows:

$$
\lambda_{A_{i}}(\nu, \alpha, \tau, \sigma, \theta)=\lambda_{A}^{(\sigma \theta)^{(\nu-1) \alpha \tau}} .
$$

Among them, $\lambda_{A}$ is the credit risk transmission rate of the counterparty in the enterprise credit-related network $A$.

Equation (1) shows that $\left(\partial \lambda_{A_{i}} / \partial \nu\right)>0$ and $\left(\partial^{2} \lambda_{A_{i}} / \partial \nu^{2}\right)<0$; thus, the credit risk contagion probability $\lambda_{A_{i}}$ of the counterparty of the corporate credit-related network has a rising characteristic of decreasing margin with the increase in the initial economic shock $v$ on the counterparty of the enterprise. Similarly, given that $\left(\partial \lambda_{A_{i}} / \partial \alpha\right)<0$ and $\left(\partial^{2} \lambda_{A_{i}} / \partial \alpha^{2}\right)<0$, the credit risk contagion probability $\lambda_{A_{i}}$ of the counterparty of the corporate credit-related network is showing a decreasing characteristic of marginal increase with the increase in the enterprise counterparty risk awareness level $\alpha$. Considering that $\left(\partial \lambda_{A_{i}} / \partial \tau\right)<0$ and $\left(\partial^{2} \lambda_{A_{i}} / \partial \tau^{2}\right)<0$, the credit risk contagion probability $\lambda_{A_{i}}$ of the counterparty of the corporate credit-related network has a decreasing characteristic of increasing margin with the increase in the counterparty riskoverflow capacity $\tau$ of the enterprise. Given that $\left(\partial \lambda_{A_{i}} / \partial \sigma\right)>0$ and $\left(\partial^{2} \lambda_{A_{i}} / \partial \sigma^{2}\right)<0$, the credit risk contagion probability $\lambda_{A_{i}}$ of the counterparty of the corporate credit-related network has a rising characteristic of decreasing margin with the increase in the leverage level $\sigma$ of the corporate counterparty. Considering that $\left(\partial \lambda_{A_{i}} / \partial \theta\right)>0$ and $\left(\partial^{2} \lambda_{A_{i}} / \partial \theta^{2}\right)<0$, the credit risk contagion probability $\lambda_{A_{i}}$ of the counterparty of the corporate credit-related network is increasing with decreasing margin with the increase in the influence $\theta$ of the counterparty of the enterprise.
3.2.2. Credit Risk Contagion Probability of Bank Network Counterparties. Credit derivative transactions, interbank lending business, and wealth management product business conducted among banks will cause credit risk to flow within the banking system. A bank default will have an impact on the balance sheet of the credit-linked bank, forming financial risks and passing the risk through business-related activities among banks [10, 21, 41, 53-55]. In addition, when some credit assets between banks and enterprises are defaulted due to the initial economic shock, the risk of default will be transmitted to the bank through the loan relationship between banks and enterprises, causing the bank to suffer a risk loss. This loss will pass through the interbank borrowing, and liquidity effects in the banking market will further spread in the banking network. The bank-enterprise credit association provides an infectious channel for the transmission of corporate credit risk to the bank. Hence, the probability of contagion of the credit risk of the interbank counterparty is affected by the risk of the contagion of the corporate counterparty credit risk to the bank counterparty $\lambda_{A \longrightarrow B}\left(0<\lambda_{A \longrightarrow B}<1\right)$ influences.

In the banking network, as the degree of interbank credit association $\varepsilon(0<\varepsilon<1)$ increases, the probability of credit risk transmission within the banking system and the speed credit risk transmission rate increase. If the bank has a comprehensive risk warning mechanism and adequate capital reserves, then the bank's own risk resistance $\omega(0<\omega<1)$ is strong and is less likely to be affected by credit risk contagion $[2,13,56,57]$. Second, if the bank's ability to process default information $\rho(0<\rho<1)$ is strong, then the ability of the bank to the credit risk screening and risk warning ability is also strong, and the probability of the bank to inhibit the spread of credit risk is great. In the bank network, some bank subjects are not proficient at processing information and have a low ability to screen and warn about credit risks. When faced with underlying risks, banks have a serious sense of panic, which will be transmitted to the major institution of neighboring banks through the credit association network under the influence of the irrational behavior of the bank. Then, the neighboring bank conveys a sense of panic to the neighboring bank, finally showing a remarkable "herd mentality" $[12,58,59]$.

According to the above analysis, a close correlation is observed between the credit risk contagion and the business correlation among banks $\varepsilon$, the bank's own risk resistance $\omega$, the bank's ability to process risk information $\rho$, and the risk contagion probability of corporate counterparty credit risk contagion to bank counterparty $\lambda_{A \longrightarrow B}$ in the bank credit correlation network. Drawing on Wang et al.'s [24] credit risk conversion model, the credit risk contagion probability model of the counterparty $i$ in the bank's credit-related network is defined as follows:

$$
\lambda_{B_{i}}\left(\varepsilon, \omega, \rho, \lambda_{A \longrightarrow B}\right)=\lambda_{A}^{1 / 2} \longrightarrow B \lambda_{B}^{\omega^{(1-\rho) \varepsilon}} .
$$

Among them, $\lambda_{B}$ is the credit risk transmission rate of the counterparty in the bank's credit-related network $B$.

Equation (2) shows that $\left(\partial \lambda_{B_{i}} / \partial \lambda_{A \longrightarrow B}\right)>0$ and $\left(\partial^{2} \lambda_{B_{i}} / \partial \lambda_{A \rightarrow B}{ }^{2}\right)<0$. Thus, the credit risk contagion 
probability $\lambda_{B}$ of the counterparty $i$ in the bank network is characterized by an increase in diminishing margin with the increase in the risk contagion probability $\lambda_{A \longrightarrow B}$ of the enterprise's counterparty credit risk infecting the bank's counterparty. Similarly, given that $\left(\partial \lambda_{B_{i}} / \partial \omega\right)<0$ and $\left(\partial^{2} \lambda_{B_{i}} / \partial \omega^{2}\right)>0$, the credit risk contagion probability $\lambda_{B_{i}}$ of the counterparty $i$ in the bank network is characterized by a decrease in diminishing margin with the increase in the risk resistance $\omega$ of the bank counterparty $i$. Given that $\left(\partial \lambda_{B_{i}} / \partial \varepsilon\right)>0$ and $\left(\partial^{2} \lambda_{B_{i}} / \partial \varepsilon^{2}\right)<0$, the credit risk contagion probability $\lambda_{B_{i}}$ of the counterparty $i$ in the bank network is characterized by an increase in diminishing margin with the increase in the business connection degree $\varepsilon$ of the bank counterparty $i$. Considering that $\left(\partial \lambda_{B_{B}} / \partial \rho\right)<0$ and $\left(\partial^{2} \lambda_{B_{i}} / \partial \rho^{2}\right)<0$, the credit risk contagion probability $\lambda_{B_{i}}$ of the counterparty $i$ in the bank network is characterized by a decrease in increasing margin with the increase in the processing capacity $\rho$ of the bank counterparty $i$ for the default information.

3.3. Construction and Analysis of Infection Model. Given that the enterprise node with the scale $k_{A}$ at $t$ is at the relative densities of $S, I$, and $R, s_{k_{A}}(t), i_{k_{A}}(t)$, and $r_{k_{A}}(t)$, respectively, meet the normalization condition: $s_{k_{A}}(t)+i_{k_{A}}(t)$ $+r_{k_{A}}(t)=1$. Similarly, given that the bank node with the scale $k_{B}$ at $t$ is at the relative densities of $S, I$, and $R, s_{k_{B}}(t)$, $i_{k_{B}}(t)$, and $r_{k_{B}}(t)$, respectively, satisfy the normalization condition: $s_{k_{B}}(t)+i_{k_{B}}(t)+r_{k_{B}}(t)=1$. Drawing on Qian et al. [34] to construct the SIR virus infection model on a complex network, as shown in Figure 2, and the analysis of the credit risk contagion mechanism of bank-enterprise counterparties above, the dynamic behavior of counterparty credit risk contagion in corporate credit-related networks can be used in differential equations of the formula:

$$
\left\{\begin{array}{l}
\frac{\mathrm{d} s_{k_{A}}(t)}{\mathrm{d} t}=-\lambda_{A} k_{A} \Theta_{1}(t) s_{k_{A}}(t)-u_{A} s_{k_{A}}(t)+\eta_{A} r_{k_{A}}(t), \\
\frac{\mathrm{d} i_{k_{A}}(t)}{\mathrm{d} t}=\lambda_{A} k_{A} \Theta_{1}(t) s_{k_{A}}(t)-\phi_{A} i_{k_{A}}(t) \\
\frac{\mathrm{d} r_{k_{A}}(t)}{\mathrm{d} t}=u_{A} s_{k_{A}}(t)+\phi_{A} i_{k_{A}}(t)-\eta_{A} r_{k_{A}}(t) .
\end{array}\right.
$$

Among them,

$$
\Theta_{1}(t)=\frac{1}{\left\langle k_{A}\right\rangle} \sum_{k_{A}^{\prime}} k_{A}^{\prime} P\left(k_{A}^{\prime}\right) i_{k_{A}}(t) .
$$

$\Theta_{1}(t)$ represents the probability of randomly selecting an edge from a healthy enterprise $S_{A}$ with a degree of $k_{A}$ to be connected with a defaulting enterprise $I_{A}$ at time $t$. Its stable value is recorded as $\Theta_{1}^{*}$ in the stable state. Equation (3) depicts the transformation relationship among the healthy, default, and bankruptcy state enterprises, where the first term on the right side of the first equation represents the general term of corporate counterparty credit risk contagion, which is proportional to the degree $k_{A}$ of corporate counterparty credit risk contagion, the rate $\lambda_{A}$ of corporate counterparty credit risk contagion, and the density $s_{k_{A}}(t)$ of healthy counterparty with the degree $k_{A}$ at time $t$. The second term means that the healthy state enterprise changes into the bankruptcy state enterprise $R_{A}$ with a probability of $u_{A}$. The third term means that the bankrupt enterprise $R_{A}$ changes into a healthy enterprise $S_{A}$ with a probability of $\eta_{A}$.

After a certain period of contagion evolution, if the scale of the healthy, default, and bankruptcy state enterprises in the enterprise credit-related network $A$ eventually becomes constant, then the enterprise credit-related network $A$ is in a stable state. The default status of the enterprise $I_{A}$ in the corporate credit association network $A$ is transmitted to the healthy bank $S_{B}$ in the bank network $B$ with a probability of $\lambda_{A \rightarrow B}$ through the bank-enterprise credit association. Therefore, the bank network $B$ has two sources of credit risk for the bank $S_{B}$ in the healthy state to become the bank $I_{B}$ in the default state: (1) In the corporate credit-related network $A$, the default state counterparty $I_{A}$ transfers the credit risk to the healthy state counterparty $S_{B}$ with a probability of $\lambda_{A \longrightarrow B}$ through the bank-enterprise credit association. (2) In the bank network $B$, the healthy bank counterparty $S_{B}$ is infected by the default bank counterparty $I_{B}$. Therefore, the dynamic behavior of counterparty credit risk contagion in the bank credit association network can be described by the following differential equations:

$$
\left\{\begin{array}{l}
\frac{\mathrm{d} s_{k_{B}}(t)}{\mathrm{d} t}=-\left[\lambda_{B} k_{B} \Theta_{2}(t)+\lambda_{A \longrightarrow B} \lambda_{A}\left\langle k_{A}\right\rangle \Theta_{1}(t)\right] \\
\cdot s_{k_{B}}(t)-u_{B} s_{k_{B}}(t)+\eta_{B} r_{k_{B}}(t), \\
\frac{\mathrm{d} i_{k_{B}}(t)}{\mathrm{d} t}=\left[\lambda_{B} k_{B} \Theta_{2}(t)+\lambda_{A \longrightarrow B} \lambda_{A}\left\langle k_{A}\right\rangle \Theta_{1}(t)\right] \\
\cdot s_{k_{B}}(t)-\phi_{B} i_{k_{B}}(t), \\
\frac{\mathrm{d} r_{k_{B}}(t)}{\mathrm{d} t}=u_{B} s_{k_{B}}(t)+\phi_{B} i_{k_{B}}(t)-\eta_{B} r_{k_{B}}(t) .
\end{array}\right.
$$

Among them,

$$
\Theta_{2}(t)=\frac{1}{\left\langle k_{B}\right\rangle} \sum_{k_{B}^{\prime}} k_{B}^{\prime} P\left(k_{B}^{\prime}\right) i_{k_{B}}(t) .
$$

$\Theta_{2}(t)$ represents the probability of randomly taking an edge from the healthy bank $S_{B}$ with a degree $k_{B}$ at the time $t$ and connecting it with the counterparty $I_{B}$ of the bank in the default state. Its stable value is recorded as $\Theta_{2}^{*}$ in the stable state. Equation (5) depicts the transformation relationship among the healthy, default, and bankruptcy state banks, where the first term on the right side of the first equation indicates that the healthy state bank $S_{B}$ has turned into the default state bank $I_{B}$ in two ways: first, this method is proportional to the degree $k_{B}$ of the bank counterparty, the rate $\lambda_{B}$ of the credit risk of the bank counterparty, and the density $s_{k_{B}}(t)$ of the healthy bank counterparty with the 
degree $k_{B}$ at $t$; second, this method is proportional to the average degree $\left\langle k_{A}\right\rangle$ of the enterprise counterparty, the rate $\lambda_{A}$ of the credit risk of the enterprise counterparty, the risk contagion rate $\lambda_{A \longrightarrow B}$ of the default state node $I_{A}$ of the enterprise credit association network infecting the credit risk to the healthy state node $S_{B}$ of the bank network, and the density $s_{k_{B}}(t)$ of the healthy bank counterparty with the degree $k_{B}$ at $t$. The second term indicates that the state healthy bank changes into the bankruptcy state bank $R_{B}$ with a probability of $u_{B}$; the third term indicates that the bankruptcy bank $R_{B}$ changes into the healthy bank $S_{B}$ with a probability of $\eta_{B}$.

After a certain period of contagion evolution, if the scale of the healthy, default, and bankrupt banks in the bank network $B$ eventually becomes constant, then the bank network $B$ is in a stable state. Therefore, when the corporate credit-related $A$ and bank $B$ networks are under stable conditions, the following mainly discusses the evolutionary characteristics of the credit risk contagion rate threshold and the scale of default.

\subsubsection{Analysis of the Threshold of Corporate Network Credit} Risk Contagion Rate and the Scale of Default. This section mainly examines the threshold of the counterparty credit risk contagion rate and the scale of default in the corporate credit-related network $A$. Notably, $i_{k_{A}}^{*}$ is the size of the defaulting enterprise with the degree of $k_{A}$ when the credit risk contagion of the enterprise's counterparty tends to be stable, and $I_{A}^{*}$ is the scale of the defaulting enterprise in the entire enterprise credit-related network $A$, so $I_{A}^{*}=\sum_{k_{A}} P\left(k_{A}\right) i_{k_{A}}^{*}$. The first two expressions of (3) equal to 0 and the normalization condition, that is,

$$
\left\{\begin{array}{l}
\frac{\mathrm{d} s_{k_{A}}(t)}{\mathrm{d} t}=0, \\
\frac{\mathrm{d} i_{k_{A}}(t)}{\mathrm{d} t}=0, \\
s_{k_{A}}(t)+i_{k_{A}}(t)+r_{k_{A}}(t)=1 .
\end{array}\right.
$$

The scale of the defaulted enterprise with the degree of $k_{A}$ can be obtained when the counterparty credit risk contagion tends to be stable in the enterprise credit association network $A$ :

$$
i_{k_{A}}^{*}=\frac{\eta_{A} \lambda_{A} k_{A} \Theta_{1}^{*}}{\eta_{A} \lambda_{A} k_{A} \Theta_{1}^{*}+\left(\lambda_{A} k_{A} \Theta_{1}^{*}+u_{A}+\eta_{A}\right) \phi_{A}} .
$$

Combining equation (8) with equation (4), we can obtain

$$
\begin{aligned}
\Theta_{1}^{*} & =\frac{1}{\left\langle k_{A}\right\rangle} \sum_{k_{A}^{\prime}} k_{A}^{\prime} P\left(k_{A}^{\prime}\right) \frac{\eta_{A} \lambda_{A} k_{A} \Theta_{1}^{*}}{\eta_{A} \lambda_{A} k_{A} \Theta_{1}^{*}+\left(\lambda_{A} k_{A} \Theta_{1}^{*}+u_{A}+\eta_{A}\right) \phi_{A}} \\
& =G\left(\Theta_{1}^{*}\right) .
\end{aligned}
$$

$\Theta_{1}^{*}=0$ is a trivial solution to equation (9). In other words, the credit risk of corporate credit-related network counterparties is not widely spread or even infected to other corporate counterparties. However, equation (9) must have a nontrivial solution $\Theta_{1}^{*}$ and satisfy $\left(\left(\mathrm{dG}\left(\Theta_{1}^{*}\right) /\right.\right.$ $\left.\left.\mathrm{d} \Theta_{1}^{*}\right) \mid \Theta_{1}^{*}=0\right) \geq 1$, and $\left(1 /\left\langle k_{A}\right\rangle\right) \sum_{k_{A}^{\prime}} k_{A}^{\prime} P\left(k_{A}^{\prime}\right) \quad\left(\eta_{A} \lambda_{A} k_{A} /\right.$ $\left.\left(u_{A}+\eta_{A}\right) \phi_{A}\right) \geq 1$ can be obtained through simplification. Therefore, the critical conditions for the credit risk contagion of counterparties in the enterprise credit-related network are as follows: $\lambda_{A}^{(\sigma \theta)^{(\nu-1) \alpha \tau}}=\left(\left\langle k_{A}\right\rangle\left(\left(u_{A} / \eta_{A}\right)+1\right) \phi_{A} /\right.$ $\left.\left\langle k_{A}^{2}\right\rangle\right)$, and the rate threshold of the credit risk contagion of the counterparty in the enterprise credit-related network is $\lambda_{A}^{*}=\left[\left\langle k_{A}\right\rangle\left(\left(u_{A} / \eta_{A}\right)+1\right) \phi_{A} /\left\langle k_{A}^{2}\right\rangle\right]^{(\sigma \theta)^{(1-v) \alpha \tau}}$, where $\left\langle k_{A}\right\rangle=$ $\sum_{k_{A}} k_{A} P_{A}\left(k_{A}\right)$ and $\left\langle k_{A}^{2}\right\rangle=\sum_{k_{A}} k_{A}^{2} P_{A}\left(k_{A}\right)$. Finally, the default enterprise scale in the enterprise credit association network $A$ is $I_{A}^{*}=\sum_{k_{A}} P_{A}\left(k_{A}\right) i_{k_{A}}^{*}=\sum_{k_{A}} P_{A}\left(k_{A}\right)\left(\eta_{A} \lambda_{A} k_{A} \Theta_{1}^{*} / \eta_{A} \lambda_{A}\right.$ $\left.k_{A} \Theta_{1}^{*}+\left(\lambda_{A} k_{A} \Theta_{1}^{*}+u_{A}+\eta_{A}\right) \phi_{A}\right)$. Among them, $\Theta_{1}^{*}$ is the nontrivial solution of equation (9), and $\Theta_{1}^{*}=$ $\left(\eta_{A} \lambda_{A} k_{A}-\left(u_{A}+\eta_{A}\right) \phi_{A} /\left(\eta_{A}+\phi_{A}\right) \lambda_{A} k_{A}\right)$.

According to the above analysis, we can see that $\left(\partial \lambda_{A}^{*} / \partial \nu\right)<0,\left(\partial^{2} \lambda_{A}^{*} / \partial \nu^{2}\right)>0,\left(\partial \lambda_{A}^{*} / \partial \alpha\right)>0,\left(\partial^{2} \lambda_{A}^{*} / \partial \alpha^{2}\right)<0$, $\left(\partial \lambda_{A}^{*} / \partial \tau\right)>0,\left(\partial^{2} \lambda_{A}^{*} / \partial \tau^{2}\right)<0,\left(\partial \lambda_{A}^{*} / \partial \sigma\right)<0,\left(\partial^{2} \lambda_{A}^{*} / \partial \sigma^{2}\right)>0$, $\left(\partial \lambda_{A}^{*} / \partial \theta_{i}\right)<0$, and $\left(\partial \lambda_{A}^{*} / \partial \theta_{i}\right)>0$. Therefore, the following proposition can be obtained.

Proposition 1. A diminishing marginal negative correlation relationship is observed between the threshold of corporate counterparty credit risk contagion $\lambda_{A}^{*}$ and corporate counterparty's initial economic shock $v$, corporate counterparty's leverage level $\sigma$, and corporate counterparty's influence $\theta_{i}$. Furthermore, a diminishing marginal positive correlation relationship is found between the threshold of corporate counterparty credit risk contagion $\lambda_{A}^{*}$, enterprise counterparty risk perception level $\alpha$, and enterprise counterparty risk capacity $\tau$.

Jackson [60] noted that random dominance is used to analyze the influence of network structure characteristics on behavioral finance. The enterprise counterparty networks $A^{\prime}$ and $A$ of the degree distribution are made of $P_{A^{\prime}}$ and $P_{A}$, respectively. Given that $G^{\prime}\left(\Theta_{1}^{*}\right)=\left(\left(\left\langle k_{A}^{2}\right\rangle /\left\langle k_{A}\right\rangle\right) \quad\left(\eta_{A} \lambda_{A}\right.\right.$ $\left.\left.\left(u_{A}+\eta_{A}\right) \phi_{A} /\left[\eta_{A} \lambda_{A} k_{A} \Theta_{1}^{*}+\left(\lambda_{A} k_{A} \Theta_{1}^{*}+u_{A}+\eta_{A}\right) \phi_{A}\right]^{2}\right)\right)>0$ and $\quad G^{\prime \prime}\left(\Theta_{1}^{*}\right)=\left(\left(\left\langle k_{A}^{2}\right\rangle /\left\langle k_{A}\right\rangle\right)\left(-\eta_{A} \lambda_{A}\left(u_{A}+\quad \eta_{A}\right) \phi_{A} 2\right.\right.$ $\left[\eta_{A} \lambda_{A} k_{A} \Theta_{1}^{*}+\left(\lambda_{A} k_{A} \Theta_{1}^{*}+u_{A}+\eta_{A}\right) \phi_{A}\right] \lambda_{A} k_{A}\left(\eta_{A}+\phi_{A}\right) / \quad\left[\eta_{A}\right.$ $\left.\left.\left.\lambda_{A} k_{A} \Theta_{1}^{*}+\left(\lambda_{A} k_{A} \Theta_{1}^{*}+u_{A}+\eta_{A}\right) \phi_{A}\right]^{4}\right)\right)<0, \quad G\left(\Theta_{1}^{*}\right) \quad$ is a monotonically increasing convex function about $\Theta_{1}^{*}$. Considering that $G(1)=\left(\left(\sum_{k_{A}^{\prime}} k_{A}^{\prime} P\left(k_{A}^{\prime}\right) /\left\langle k_{A}\right\rangle\right)\left(\eta_{A} \lambda_{A} k_{A} / \eta_{A} \lambda_{A} k_{A}+\right.\right.$ $\left.\left.\left(\lambda_{A} k_{A}+u_{A}+\eta_{A}\right) \phi_{A}\right)\right)<\left(\left(\sum_{k_{A}^{\prime}} k_{A}^{\prime} P\left(k_{A}^{\prime}\right) /\left\langle k_{A}\right\rangle\right)\left(\eta_{A} \lambda_{A} k_{A} / \eta_{A} \lambda_{A}\right.\right.$ $\left.\left.k_{A}\right)\right)=1$ and $G(0)=0, G\left(\Theta_{1}^{*}\right)$ has at least one fixed point on $[0,1]$. When $\lambda_{A}>\lambda_{A}^{*}$, the credit risk of the enterprise's counterparty spreads to the entire enterprise's credit association network, and this network reaches an equilibrium state, at this time, $G^{\prime}\left(\Theta_{1}^{*}\right) \mid \Theta_{1}^{*}=0>1$. Therefore, $G\left(\Theta_{1}^{*}\right)=\left(1 /\left\langle k_{A}\right\rangle\right) \sum_{k_{A}^{\prime}} k_{A}^{\prime}$ $P\left(k_{A}^{\prime}\right)\left(\eta_{A} \lambda_{A} k_{A} \Theta_{1}^{*} / \eta_{A} \lambda_{A} k_{A} \Theta_{1}^{*}+\left(\lambda_{A} k_{A} \Theta_{1}^{*}+u_{A}+\eta_{A}\right) \phi_{A}\right)$, and a unique equilibrium point is observed on $[0,1]$ and $\Theta_{1}^{*}>0$. 
Theorem 1. When $\lambda_{A}>\lambda_{A}^{*}$, under other conditions unchanged, if the average degree of corporate counterparty credit-related network $A^{\prime}$ is greater than the average degree of the corporate counterparty credit-related network $A$, then (1) the equilibrium value $\Theta_{1}^{*}$ of the counterparty credit risk diffusion rate in network $A^{\prime}$ is greater than the equilibrium value $\Theta_{1}^{*}$ of the counterparty credit risk diffusion rate in network $A$ and (2) the diffusion scale $I_{A}^{* \prime}$ of the counterparty credit risk in network $A^{\prime}$ is greater than the diffusion scale $I_{A}^{*}$ of the counterparty credit risk in network A.

Proof: Supposing (1) that the original theorem is untrue, the average degree of corporate counterparty credit-related network $A^{\prime}$ is greater than the average degree of corporate counterparty credit-related network $A$, and $\Theta_{1}^{*} \leq \Theta_{1}^{*}$; that is, $\Theta_{1}^{*} \geq \Theta_{1}^{*^{\prime}}=G\left(\Theta_{1}^{*^{\prime}}\right)$. When $\lambda_{A}>\lambda_{A}^{*}$, the equilibrium value $\Theta_{1}^{*}$ of the credit risk diffusion rate of the counterparty of the enterprise's credit-linked network is unique, and $\Theta_{1}^{*}>0$. If $H(k)=\eta_{A} \lambda_{A} k \Theta_{1}^{*} /\langle k\rangle\left[\eta_{A} \lambda_{A} k \Theta_{1}^{*}+\left(\lambda_{A} k \Theta_{1}^{*}+u_{A}+\eta_{A}\right) \phi_{A}\right]$, then $(\partial H(k) / \partial k)=\left(\eta_{A} \lambda_{A} \Theta_{1}^{*}\langle k\rangle\left(u_{A}+\eta_{A}\right) \phi_{A} /\left\{\langle k\rangle\left[\eta_{A} \lambda_{A}\right.\right.\right.$ $\left.\left.\left.k \Theta_{1}^{*}+\left(\lambda_{A} k \Theta_{1}^{*}+u_{A}+\eta_{A}\right) \phi_{A}\right]\right\}^{2}\right)>0$. Therefore, $H(k)$ is a monotonically increasing function of $k$. According to the theory of random dominance of the network, the average degree $k_{A^{\prime}}>k_{A} \Longrightarrow P_{A^{\prime}}$ is strictly first-order random dominance $P_{A} \Longrightarrow$ because for any $\Theta^{*}>0, \quad G\left(\Theta^{* \prime}\right)>$ $G\left(\Theta^{*}\right) \Longrightarrow$ exists, and for any $\Theta_{1}^{*}>0, G\left(\Theta_{1}^{*}\right)>G\left(\Theta_{1}^{*}\right)$ exists. Hence, $\Theta_{1}^{*} \geq G\left(\Theta_{1}^{*}\right)>G\left(\Theta_{1}^{*}\right)$. This notion contradicts $\Theta_{1}^{*}=G\left(\Theta_{1}^{*}\right)$. Therefore, the original conclusion (1) holds.

Conclusion (1) shows that when the average degree of the enterprise's counterparty credit-related network $A^{\prime}$ is greater than the average degree of the enterprise's counterparty credit-related network $A, \Theta_{1}^{*}>\Theta_{1}^{*}$ evidently $i_{k_{A}}^{*}=$ $\eta_{A} \lambda_{A} k_{A} \Theta_{1}^{*} / \eta_{A} \lambda_{A} k_{A} \Theta_{1}^{*}+\left(\lambda_{A} k_{A} \Theta_{1}^{*}+u_{A}+\eta_{A}\right) \phi_{A}$ is a monotone increasing function about $\Theta_{1}^{*}$. Therefore, for any $k>0, i_{k_{A^{\prime}}}^{*}>i_{k_{A}}^{*}$ occurs. In addition, $\sum_{k_{A}} i_{k_{A^{\prime}}}^{*}>\sum_{k_{A}} i_{k_{A}}^{*}$; therefore, $\quad \sum_{k_{A}} p\left(k_{A^{\prime}}\right) i_{A_{A^{\prime}}}^{*}>\sum_{k_{A}} p\left(k_{A^{\prime}}\right) i_{k_{A}}^{*} ; \quad$ that is, $I_{A^{\prime}}^{*}>$ $\sum_{k_{A}} p\left(k_{A^{\prime}}\right) i_{k_{A}}^{*}$. According to the theory of network random dominance, the average degree $k_{A^{\prime}}>k_{A} \Rightarrow P_{A^{\prime}}$ is strictly a first-order random dominance $P_{A} \Rightarrow \sum_{k_{A}} p\left(k_{A^{\prime}}\right) i_{k_{A}}^{*}>$ $\sum_{k_{A}} P\left(k_{A}\right) i_{k_{A}}^{*}$. Therefore, $I_{A^{\prime}}^{*}>\sum_{k_{A}} P\left(k_{A^{\prime}}\right) i_{k_{A}}^{*}>\sum_{k_{A}} P\left(k_{A}\right) i_{k_{A}}^{*}=$ $I_{A}^{*}$. Hence, conclusion (2) was proved.

3.3.2. Analysis of the Threshold of Bank Network Credit Risk Contagion Rate and the Scale of Default. This section mainly examines the threshold of the credit default risk contagion rate of the counterparty in the bank's credit-related network $B$ and the scale of default. Notably, $i_{k_{B}}^{*}$ is the size of the default bank with the degree of $k_{B}$ when the credit risk contagion of the bank's counterparty tends to be stable, and $I_{B}^{*}$ be the scale of the default bank when the credit risk contagion of the counterparty in the entire bank's creditrelated network is stable.

Given that the first two expressions of (5) equal 0 , the normalization condition is

$$
\left\{\begin{array}{l}
\frac{\mathrm{d} s_{k_{B}}(t)}{\mathrm{d} t}=0, \\
\frac{\mathrm{d} i_{k_{B}}(t)}{\mathrm{d} t}=0, \\
s_{k_{B}}(t)+i_{k_{B}}(t)+r_{k_{B}}(t)=1 .
\end{array}\right.
$$

The scale of the default bank with a degree of $k_{B}$ can be obtained when the counterparty credit risk contagion tends to be stable in the bank's credit-related network $B$ :

$$
i_{k_{B}}^{*}=\frac{\eta_{B}\left[\lambda_{B} k_{B} \Theta_{2}^{*}+\lambda_{A \longrightarrow B} \lambda_{A}\left\langle k_{A}\right\rangle \Theta_{1}^{*}\right]}{\eta_{B}\left[\lambda_{B} k_{B} \Theta_{2}^{*}+\lambda_{A \longrightarrow B} \lambda_{A}\left\langle k_{A}\right\rangle \Theta_{1}^{*}\right]+\phi_{B}\left[\eta_{B}+\lambda_{B} k_{B} \Theta_{2}^{*}+\lambda_{A \longrightarrow B} \lambda_{A}\left\langle k_{A}\right\rangle \Theta_{1}^{*}+u_{B}\right]} .
$$

Combining equation (9) with equation (6), we can obtain

$$
\Theta_{2}^{*}=\frac{1}{\left\langle k_{B}\right\rangle} \sum_{k_{B}^{\prime}} k_{B}^{\prime} P\left(k_{B}^{\prime}\right) \frac{\eta_{B}\left[\lambda_{B} k_{B} \Theta_{2}^{*}+\lambda_{A \longrightarrow B} \lambda_{A}\left\langle k_{A}\right\rangle \Theta_{1}^{*}\right]}{\eta_{B}\left[\lambda_{B} k_{B} \Theta_{2}^{*}+\lambda_{A \longrightarrow B} \lambda_{A}\left\langle k_{A}\right\rangle \Theta_{1}^{*}\right]+\phi_{B}\left[\eta_{B}+\lambda_{B} k_{B} \Theta_{2}^{*}+\lambda_{A \longrightarrow B} \lambda_{A}\left\langle k_{A}\right\rangle \Theta_{1}^{*}+u_{B}\right]}=G\left(\Theta_{2}^{*}\right) .
$$

$\Theta_{2}^{*}=0$ is a trivial solution of equation (12); in other words, the credit risk of bank credit-related network counterparties is not widely spread or even infected to other bank counterparties. However, equation (12) must have a nontrivial solution and satisfy $\left.\left(\mathrm{dG}\left(\Theta_{2}^{*}\right) / \mathrm{d}_{2}^{*}\right)\right|_{\Theta_{2}^{*}=0} \geq 1$, and $\left(\left\langle k_{B}^{2}\right\rangle /\left\langle k_{B}\right\rangle\right)\left(\eta_{B} \lambda_{B} \phi_{B}\left(\eta_{B}+u_{B}\right) / \eta_{B} \lambda_{A \longrightarrow B} \lambda_{A}\left\langle k_{A}\right\rangle \Theta_{1}^{*}+\right.$ $\left.\phi_{B}\left(\eta_{B}+\lambda_{A \longrightarrow B} \lambda_{A}\left\langle k_{A}\right\rangle \Theta_{1}^{*}+u_{B}\right)^{2}\right) \geq 1$ can be obtained after simplification. Therefore, the critical condition for obtaining the credit risk of the counterparty of the bank's credit-related network is $\lambda_{A}^{1 / 2} \longrightarrow{ }_{B} \lambda_{B}^{\omega^{(1-\rho) \varepsilon}}=\left\langle k_{B}\right\rangle\left[\eta_{B} \lambda_{A} \longrightarrow\right.$ $\left.B \lambda_{A}\left\langle k_{A}\right\rangle \Theta_{1}^{*}+\phi_{B}\left(\eta_{B}+\lambda_{A \longrightarrow B} \lambda_{A}\left\langle k_{A}\right\rangle \Theta_{1}^{*}+u_{B}\right)^{2}\right] /\left\langle k_{B}^{2}\right\rangle \eta_{B} \phi$ ${ }_{B}\left(\eta_{B}+u_{B}\right)$, and the rate threshold of the credit risk contagion of the counterparty in the bank credit-related network is $\lambda_{B}^{*}=\left[\lambda_{A}^{-1 / 2} \longrightarrow{ }_{B}\left\langle k_{B}\right\rangle\left[\eta_{B} \lambda_{A \longrightarrow B} \lambda_{A}\left\langle k_{A}\right\rangle \Theta_{1}^{*}+\right.\right.$ 
$\left.\phi_{B}\left(\eta_{B}+\lambda_{A \longrightarrow B} \lambda_{A} \quad\left\langle k_{A}\right\rangle \Theta_{1}^{*}+u_{B}\right)^{2}\right] / \quad\left\langle k_{B}^{2}\right\rangle \eta_{B} \phi_{B}\left(\eta_{B}+\right.$
$\left.\left.u_{B}\right)\right]^{\omega^{(\rho-1) \varepsilon}}$
Among them, $\left\langle k_{B}\right\rangle=\sum_{k_{B}} k_{B} P_{B}\left(k_{B}\right)$ and $\left\langle k_{B}^{2}\right\rangle=$ $\sum_{k_{B}} k_{B}^{2} P_{B}\left(k_{B}\right)$. Finally, the default bank scale in the bank credit association network $B$ is

$$
I_{B}^{*}=\sum_{k_{B}} P_{B}\left(k_{B}\right) i_{k_{B}}^{*}=\sum_{k_{B}} P_{B}\left(k_{B}\right) \frac{\eta_{B}\left[\lambda_{B} k_{B} \Theta_{2}^{*}+\lambda_{A \longrightarrow B} \lambda_{A}\left\langle k_{A}\right\rangle \Theta_{1}^{*}\right]}{\eta_{B}\left[\lambda_{B} k_{B} \Theta_{2}^{*}+\lambda_{A \longrightarrow B} \lambda_{A}\left\langle k_{A}\right\rangle \Theta_{1}^{*}\right]+\phi_{B}\left[\eta_{B}+\lambda_{2} k_{B} \Theta_{2}^{*}+\lambda_{A \longrightarrow B} \lambda_{A}\left\langle k_{A}\right\rangle \Theta_{1}^{*}+u_{B}\right]},
$$

where $\Theta_{2}^{*}$ is the nontrivial solution of equation (12), $\Theta_{2}^{*}=-a+\sqrt{b^{2}-c} / 2\left(\eta_{A}+\phi_{A}\right)\left(\eta_{B}+\phi_{B}\right) \lambda_{B} k_{B}, \quad a=b=\left(\eta_{A}+\right.$ $\left.\phi_{A}\right) \phi_{B}\left(\eta_{B}+u_{B}\right)-\left(\eta_{B}+\phi_{B}\right) \lambda_{A \longrightarrow B}\left(u_{A}+\eta_{A}\right) \phi_{A}+\left(\eta_{B}+\phi_{B}\right)$ $\lambda_{A \longrightarrow B} \eta_{A} \lambda_{A} k_{A}-\left(\eta_{A}+\phi_{A}\right) \eta_{B} \lambda_{B} k_{B}$, and $c=4\left(\eta_{A}+\phi_{A}\right)$ $\left(\eta_{B}+\phi_{B}\right) \lambda_{B} k_{B} \eta_{B} \lambda_{3}\left[\eta_{A} \lambda_{A} k_{A}-\left(u_{A}+\eta_{A}\right) \phi_{A}\right]$.

According to the infection model of Section 3.3, we can know $\quad\left(\partial \lambda_{B}^{*} / \partial \varepsilon\right)<0, \quad\left(\partial^{2} \lambda_{B}^{*} / \partial \varepsilon^{2}\right)>0, \quad\left(\partial \lambda_{B}^{*} / \partial \omega\right)>0$, $\left(\partial^{2} \lambda_{B}^{*} / \partial \omega^{2}\right)>0$, $\left(\partial \lambda_{B}^{*} / \partial \rho\right)>0$, and $\left(\partial^{2} \lambda_{B}^{*} / \partial \rho^{2}\right)>0$. Therefore, the following proposition can be obtained:

Proposition 2. A diminishing marginal negative correlation relationship is observed between the threshold of bank counterparty credit risk contagion $\lambda_{B}^{*}$ and the degree of business correlation $\varepsilon$ of the bank's counterparty. Moreover, an increasing marginal positive correlation relationship is found between the threshold of bank counterparty credit risk contagion $\lambda_{B}^{*}$, the risk resistance of the bank's counterparty $\omega$, and the ability of the bank's counterparty to process risk information $\rho$.

Case 1. Under the established structure of the bank credit association network, the impact characteristics of different corporate credit-related network structures on the bank network credit risk contagion rate threshold and the scale of default are analyzed.

$k_{B}$ is constant, and the degree distribution $P_{B}$ of the bank's credit-related network is also constant. Given that

$$
\begin{aligned}
G^{\prime}\left(\Theta_{2}^{*}\right) & =\frac{\phi_{B}\left[\eta_{B}+\lambda_{B} k_{B} \Theta_{2}^{*}+\lambda_{A \longrightarrow B} \lambda_{A}\left\langle k_{A}\right\rangle \Theta_{1}^{*}+u_{B}\right]}{\left[\eta_{B}\left(\lambda_{B} k_{B} \Theta_{2}^{*}+\lambda_{A \longrightarrow B} \lambda_{A}\left\langle k_{A}\right\rangle \Theta_{1}^{*}\right)+\phi_{B}\left(\eta_{B}+\lambda_{B} k_{B} \Theta_{2}^{*}+\lambda_{A \longrightarrow B} \lambda_{A}\left\langle k_{A}\right\rangle \Theta_{1}^{*}+u_{B}\right)\right]^{2}}>0, \\
G^{\prime \prime}\left(\Theta_{2}^{*}\right) & =\frac{\phi_{B} \lambda_{B} k_{B}-\phi_{B}\left[\eta_{B}+\lambda_{B} k_{B} \Theta_{2}^{*}+\lambda_{A \longrightarrow B} \lambda_{A}\left\langle k_{A}\right\rangle \Theta_{1}^{*}+u_{B}\right] 4\left[\eta_{B}\left(\lambda_{B} k_{B} \Theta_{2}^{*}+\lambda_{A \longrightarrow B} \lambda_{A}\left\langle k_{A}\right\rangle \Theta_{1}^{*}\right)+\phi_{B}\left(\eta_{B}+\lambda_{B} k_{B} \Theta_{2}^{*}+\lambda_{A \longrightarrow B} \lambda_{A}\left\langle k_{A}\right\rangle \Theta_{1}^{*}+u_{B}\right)\right]}{\left[\eta_{B}\left(\lambda_{B} k_{B} \Theta_{2}^{*}+\lambda_{A} \longrightarrow{ }_{B} \lambda_{A}\left\langle k_{A}\right\rangle \Theta_{1}^{*}\right)+\phi_{B}\left(\eta_{B}+\lambda_{B} k_{B} \Theta_{2}^{*}+\lambda_{A} \longrightarrow{ }_{B} \lambda_{A}\left\langle k_{A}\right\rangle \Theta_{1}^{*}+u_{B}\right)\right]^{2}}<,
\end{aligned}
$$

$G\left(\Theta_{2}^{*}\right)$ is a monotonically increasing convex function about $\Theta_{2}^{*}$. Considering that

$$
\begin{aligned}
& G(1)=\frac{\sum_{k_{B}^{\prime}} k_{B}^{\prime} P\left(k_{B}^{\prime}\right)}{\left\langle k_{B}\right\rangle} \frac{\eta_{B}\left[\lambda_{B} k_{B}+\lambda_{A \longrightarrow B} \lambda_{A}\left\langle k_{A}\right\rangle \Theta_{1}^{*}\right]}{\eta_{B}\left[\lambda_{B} k_{B}+\lambda_{A \longrightarrow B} \lambda_{A}\left\langle k_{A}\right\rangle \Theta_{1}^{*}\right]+\phi_{B}\left[\eta_{B}+\lambda_{B} k_{B}+\lambda_{A \longrightarrow B} \lambda_{A}\left\langle k_{A}\right\rangle \Theta_{1}^{*}+u_{B}\right]} \\
& <\frac{\sum_{k_{B}^{\prime}} k_{B}^{\prime} P\left(k_{B}^{\prime}\right)}{\left\langle k_{B}\right\rangle} \frac{\eta_{B}\left[\lambda_{B} k_{B}+\lambda_{A \longrightarrow B} \lambda_{A}\left\langle k_{A}\right\rangle \Theta_{1}^{*}\right]}{\eta_{B}\left[\lambda_{B} k_{B}+\lambda_{A \longrightarrow B} \lambda_{A}\left\langle k_{A}\right\rangle \Theta_{1}^{*}\right]}=1,
\end{aligned}
$$

$G(0)=0$. Therefore, $G\left(\Theta_{2}^{*}\right)$ has at least one fixed point on $[0,1]$. When $\lambda_{B}>\lambda_{B}^{*}$, the credit risk of the bank's counterparty spreads to the entire bank's credit-related network, and this network reaches an equilibrium state, at this time, $\left.G^{\prime}\left(\Theta_{1}^{*}\right)\right|_{\Theta_{1}^{*}=0}>1$. Therefore,

$$
G\left(\Theta_{2}^{*}\right)=\frac{1}{\left\langle k_{B}\right\rangle} \sum_{k_{B}^{\prime}} k_{B}^{\prime} P\left(k_{B}^{\prime}\right) \frac{\eta_{B}\left[\lambda_{B} k_{B} \Theta_{2}^{*}+\lambda_{A \longrightarrow B} \lambda_{A}\left\langle k_{A}\right\rangle \Theta_{1}^{*}\right]}{\eta_{B}\left[\lambda_{B} k_{B} \Theta_{2}^{*}+\lambda_{A \longrightarrow B} \lambda_{A}\left\langle k_{A}\right\rangle \Theta_{1}^{*}\right]+\phi_{B}\left[\eta_{B}+\lambda_{B} k_{B} \Theta_{2}^{*}+\lambda_{A \longrightarrow B} \lambda_{A}\left\langle k_{A}\right\rangle \Theta_{1}^{*}+u_{B}\right]} .
$$

has a unique equilibrium point on $[0,1]$, and $\Theta_{2}^{*}>0$. 
Theorem 2. When $\lambda_{B}>\lambda_{B}^{*}$, under other conditions unchanged and under the established bank network structure, if the average degree of corporate credit-related network $A^{\prime}$ is greater than the average degree of corporate credit-related network $A$, then (1) the equilibrium value $\Theta_{1}^{*}$ of the counterparty credit risk diffusion rate in the bank creditrelated network $B^{\prime}$ is greater than that $\Theta_{2}^{*}$ of the counterparty credit risk diffusion rate in the bank credit-related network $B$ and (2) the scale $I_{B}^{*}$ ' of the counterparty credit risk diffusion in the bank credit-related network $B^{\prime}$ is larger than that $I_{B}^{*}$ of the counterparty credit risk diffusion in the bank credit-related network $B$.

Proof: Considering (1) that the original theorem is not true, the average degree of corporate counterparty credit-related network $A^{\prime}$ is greater than the average degree of corporate counterparty credit-related network $A$, and $\Theta_{2}^{*} \leq \Theta_{2}^{*}$; that is, $\Theta_{2}^{*} \geq \Theta_{2}^{*^{\prime}}=G\left(\Theta_{2}^{*^{\prime}}\right)$. When $\lambda_{B}>\lambda_{B}^{*}$, the equilibrium value $\Theta_{2}^{*}$ of the credit risk diffusion rate of the counterparty of the bank's credit-linked network is unique, and $\Theta_{2}^{*}>0$. If $H(\langle k\rangle)=\eta_{B}\left[\lambda_{B} k_{B} \Theta_{2}^{*}+\lambda_{A \longrightarrow B} \lambda_{A}\langle k\rangle \Theta_{1}^{*}\right] /\left\langle k_{B}\right\rangle \eta_{B}\left[\lambda_{B} k_{B} \Theta_{2}^{*}+\right.$ $\left.\lambda_{A \longrightarrow B} \lambda_{A}\langle k\rangle \Theta_{1}^{*}\right]+\phi_{B}\left[\eta_{B}+\lambda_{B} k_{B} \Theta_{2}^{*}+\lambda_{A \longrightarrow B} \lambda_{A}\langle k\rangle \Theta_{1}^{*}+u_{B}\right]$, then $(\partial H(\langle k\rangle) / \partial\langle k\rangle)=\left(\phi_{B} \lambda_{B} k_{B} \Theta_{2}^{*}\left[1-\eta_{B} \lambda_{A \longrightarrow B} \lambda_{A} \Theta_{1}^{*}\right]+\right.$ $\lambda_{A \longrightarrow B} \lambda_{A}\langle k\rangle \Theta_{1}^{*} \phi_{B}\left[1-\eta_{B} \lambda_{A \longrightarrow B} \lambda_{A} \Theta_{1}^{*}\right]+\phi_{B}\left(\eta_{B}+u_{B}\right) /\left\{\left\langle k_{B}\right\rangle\right.$ $\eta_{B}\left[\lambda_{B} k_{B} \Theta_{2}^{*}+\lambda_{A \longrightarrow B} \lambda_{A}\langle k\rangle \Theta_{1}^{*}\right]+\phi_{B}\left[\eta_{B}+\lambda_{B} k_{B} \quad \Theta_{2}^{*}+\lambda_{A \longrightarrow B}\right.$ $\left.\left.\left.\lambda_{A}\langle k\rangle \Theta_{1}^{*}+u_{B}\right]\right\}^{2}\right)>0$. Therefore, $H(\langle k\rangle)$ is a monotonically increasing function of $\langle k\rangle$. According to the theory of random dominance of the network, the average degree $\left\langle k_{A^{\prime}}\right\rangle>\left\langle k_{A}\right\rangle \Longrightarrow \sum_{k_{B}} H\left(\left\langle k_{A^{\prime}}\right\rangle\right) p\left(k_{B}\right)>\sum_{k_{B}} H\left(\left\langle k_{A}\right\rangle\right) p\left(k_{B}\right)$. Therefore, $\forall \Theta^{*}>0, \quad G\left(\Theta^{*^{\prime}}\right)>G\left(\Theta^{*}\right) \Longrightarrow \forall \Theta_{2}^{*}>0$, and $G\left(\Theta_{2}^{*^{\prime}}\right)>G\left(\Theta_{2}^{*}\right)$. Thus, $\Theta_{2}^{*} \geq G\left(\Theta_{2}^{*^{\prime}}\right)>G\left(\Theta_{2}^{*}\right)$. This finding contradicts $\Theta_{2}^{*}=G\left(\Theta_{2}^{*}\right)$. Therefore, the original conclusion (1) holds.

Conclusion (1) shows that when the average degree of enterprise credit-related network $A^{\prime}$ is greater than the average degree of enterprise credit-related network $A$, $\Theta_{2}^{*^{\prime}}>\Theta_{2}^{*} \cdot i_{k_{B}}^{*}=\eta_{B}\left[\lambda_{2} k_{B} \Theta_{2}^{*}+\lambda_{A \longrightarrow B} \lambda_{A}\left\langle k_{A}\right\rangle \Theta_{1}^{*}\right] / \eta_{B}\left[\lambda_{B} k_{B}\right.$ $\left.\Theta_{2}^{*}+\lambda_{A \longrightarrow B} \lambda_{A}\left\langle k_{A}\right\rangle \Theta_{1}^{*}\right]+\phi_{B}\left[\eta_{B}+\lambda_{B} k_{B} \Theta_{2}^{*}+\lambda_{A \longrightarrow B} \lambda_{A}\right.$ $\left\langle k_{A}\right\rangle \Theta_{1}^{*}+u_{B}$ ] is a monotonically increasing function of $\Theta_{2}^{*}$. Therefore, for the average degree of the credit network $\left\langle k_{A^{\prime}}\right\rangle>\left\langle k_{A}\right\rangle, \quad i_{k_{B^{\prime}}}^{*}>i_{k_{B}}^{*}$ and $\sum_{k_{B}} i_{k_{B^{\prime}}}^{*}>\sum_{k_{B}} i_{k_{B}}^{*}$ exist, so $\sum_{k_{B}} p\left(k_{B^{\prime}}\right) i_{k_{B^{\prime}}}^{*}>\sum_{k_{B}} p\left(k_{B}\right) i_{k_{B}}^{*}$; that is, $I_{B^{\prime}}^{*}>I_{B}^{*}$. Therefore, the original conclusion (2) is established.

Case 2. Under the established structure of the corporate credit-related network, the impact characteristics of different bank network structures on the bank network credit risk contagion rate threshold and the scale of default are analyzed.

If $k_{A}$ remains unchanged, then the degree distributions of network $B^{\prime}$ composed of bank counterparties and network $B$ composed of bank counterparties are $P_{B^{\prime}}$ and $P_{B}$, respectively. Considering that

$$
\begin{aligned}
G^{\prime}\left(\Theta_{2}^{*}\right) & =\frac{\phi_{B}\left[\eta_{B}+\lambda_{B} k_{B} \Theta_{2}^{*}+\lambda_{A \longrightarrow B} \lambda_{A}\left\langle k_{A}\right\rangle \Theta_{1}^{*}+u_{B}\right]}{\left[\eta_{B}\left(\lambda_{B} k_{B} \Theta_{2}^{*}+\lambda_{A \longrightarrow B} \lambda_{A}\left\langle k_{A}\right\rangle \Theta_{1}^{*}\right)+\phi_{B}\left(\eta_{B}+\lambda_{B} k_{B} \Theta_{2}^{*}+\lambda_{A \longrightarrow B} \lambda_{A}\left\langle k_{A}\right\rangle \Theta_{1}^{*}+u_{B}\right)\right]^{2}}>0, \\
G^{\prime \prime}\left(\Theta_{2}^{*}\right) & =\frac{\phi_{B} \lambda_{B} k_{B}-\phi_{B}\left[\eta_{B}+\lambda_{B} k_{B} \Theta_{2}^{*}+\lambda_{A \longrightarrow B} \lambda_{A}\left\langle k_{A}\right\rangle \Theta_{1}^{*}+u_{B}\right] 4\left[\eta_{B}\left(\lambda_{2} k_{B} \Theta_{2}^{*}+\lambda_{A \longrightarrow B} \lambda_{A}\left\langle k_{A}\right\rangle \Theta_{1}^{*}\right)+\phi_{B}\left(\eta_{B}+\lambda_{B} k_{B} \Theta_{2}^{*}+\lambda_{A \longrightarrow B} \lambda_{A}\left\langle k_{A}\right\rangle \Theta_{1}^{*}+u_{B}\right)\right]}{\left[\eta_{B}\left(\lambda_{B} k_{B} \Theta_{2}^{*}+\lambda_{A \longrightarrow B} \lambda_{A}\left\langle k_{A}\right\rangle \Theta_{1}^{*}\right)+\phi_{B}\left(\eta_{B}+\lambda_{B} k_{B} \Theta_{2}^{*}+\lambda_{A} \longrightarrow{ }_{B} \lambda_{A}\left\langle k_{A}\right\rangle \Theta_{1}^{*}+u_{B}\right)\right]^{2}}<0
\end{aligned}
$$

$G\left(\Theta_{2}^{*}\right)$ is a monotonically increasing convex function about $\Theta_{2}^{*}$. Considering that

$$
\begin{aligned}
G(1) & =\frac{\sum_{k_{B}^{\prime}} k_{B}^{\prime} P\left(k_{B}^{\prime}\right)}{\left\langle k_{B}\right\rangle} \frac{\eta_{B}\left[\lambda_{B} k_{B}+\lambda_{A \longrightarrow B} \lambda_{A}\left\langle k_{A}\right\rangle \Theta_{1}^{*}\right]}{\eta_{B}\left[\lambda_{B} k_{B}+\lambda_{A \longrightarrow B} \lambda_{A}\left\langle k_{A}\right\rangle \Theta_{1}^{*}\right]+\phi_{B}\left[\eta_{B}+\lambda_{B} k_{B}+\lambda_{A \longrightarrow B} \lambda_{A}\left\langle k_{A}\right\rangle \Theta_{1}^{*}+u_{B}\right]} \\
& <\frac{\sum_{k_{B}^{\prime}} k_{B}^{\prime} P\left(k_{B}^{\prime}\right)}{\left\langle k_{B}\right\rangle} \frac{\eta_{B}\left[\lambda_{B} k_{B}+\lambda_{A \longrightarrow B} \lambda_{A}\left\langle k_{A}\right\rangle \Theta_{1}^{*}\right]}{\eta_{B}\left[\lambda_{B} k_{B}+\lambda_{A \longrightarrow B} \lambda_{A}\left\langle k_{A}\right\rangle \Theta_{1}^{*}\right]}=1,
\end{aligned}
$$

$G(0)=0$. Therefore, $G\left(\Theta_{2}^{*}\right)$ has at least one fixed point on $[0,1]$. When $\lambda_{B}>\lambda_{B}^{*}$, the credit risk of the bank's counterparty spreads to the entire bank's credit-related network, and this network reaches a balanced state. At this time, $\left.G^{\prime}\left(\Theta_{2}^{*}\right)\right|_{\Theta_{2}^{*}=0}>1$. Therefore, $G\left(\Theta_{2}^{*}\right)=\left(1 /\left\langle k_{B}\right\rangle\right) \quad \sum_{k_{B}^{\prime}} k_{B}^{\prime} P$ $\left(k_{B}^{\prime}\right) \eta_{B}\left[\lambda_{B} k_{B} \Theta_{2}^{*}+\lambda_{A \longrightarrow B} \lambda_{A}\left\langle k_{A}\right\rangle \Theta_{1}^{*}\right] / \eta_{B}\left[\lambda_{B} k_{B} \Theta_{2}^{*}+\lambda_{A \longrightarrow B} \lambda_{A}\right.$ $\left.\left\langle k_{A}\right\rangle \Theta_{1}^{*}\right]+\phi_{B}\left[\eta_{B}+\lambda_{B} k_{B} \Theta_{2}^{*}+\lambda_{A \longrightarrow B} \lambda_{A}\left\langle k_{A}\right\rangle \Theta_{1}^{*}+u_{B}\right]$ has a unique equilibrium point on $[0,1]$, and $\Theta_{2}^{*}>0$.
Theorem 3. When $\lambda_{B}>\lambda_{B}^{*}$, under other conditions unchanged and under the established corporate credit-related network structure, if the average degree of bank credit-related network $B^{\prime}$ is greater than the average degree of bank creditrelated network $B$, then (1) the equilibrium value $\Theta_{2}^{*}$ of the counterparty credit risk diffusion rate in network $B^{\prime}$ is greater than that $\Theta_{2}^{*}$ of the counterparty credit risk diffusion rate in network $B$ and (2) the scale $I_{B}^{*}$ of counterparty credit risk 
diffusion in network $B^{\prime}$ is larger than that $I_{B}^{*}$ of counterparty credit risk diffusion in network $B$.

Proof: Considering that (1) is not true, the average degree of network $B^{\prime}$ formed by bank transaction counterparts is greater than the average degree of network $B$ formed by bank transaction counterparts, and $\Theta_{2}^{*} \leq \Theta_{2}^{*}$; that is, $\Theta_{2}^{*} \geq \Theta_{2}^{*^{\prime}}=G\left(\Theta_{2}^{*^{\prime}}\right)$. At that time, $\lambda_{B}>\lambda_{B}^{*}$, the equilibrium value $\Theta_{2}^{*}$ of the credit risk diffusion rate of the bank's network counterparty is unique, and $\Theta_{2}^{*}>0$. If $H(k)=$ $\eta_{B}\left[\lambda_{B} k \Theta_{2}^{*}+\lambda_{A \longrightarrow B} \lambda_{A}\left\langle k_{A}\right\rangle \Theta_{1}^{*}\right] /\left\langle k_{B}\right\rangle \eta_{B}\left[\lambda_{B} k \Theta_{2}^{*}+\lambda_{A \longrightarrow B} \lambda_{A}\right.$ $\left.\left\langle k_{A}\right\rangle \Theta_{2}^{*}\right]+\phi_{B} \quad\left[\eta_{B}+\lambda_{B} k \quad \Theta_{2}^{*}+\lambda_{A \longrightarrow B} \lambda_{A}\left\langle k_{A}\right\rangle \Theta_{1}^{*}+u_{B}\right]$, then $\left\{\partial H(k) / \partial k=\left(\phi_{B}\left(\eta_{B}+u_{B}\right)+\phi_{B} \lambda_{B} k \Theta_{2}^{*} \quad\left(1-\eta_{B}\right)+\right.\right.$ $\phi_{B} \lambda_{A \longrightarrow B} \lambda_{A}\left\langle k_{A}\right\rangle \Theta_{1}^{*}\left[1-\eta_{B} \lambda_{B} \Theta_{2}^{*}\right] / \quad\left\{\left\langle k_{B}\right\rangle \eta_{B}\left[\lambda_{B} k \Theta_{2}^{*}+\lambda\right.\right.$ $\left.{ }_{A \longrightarrow B} \lambda_{A}\left\langle k_{A}\right\rangle \Theta_{1}^{*}\right]+\phi_{B}\left[\eta_{B}+\lambda_{B} k \Theta_{2}^{*}+\lambda_{A \longrightarrow B} \lambda_{A}\left\langle k_{A}\right\rangle \Theta_{1}^{*}+\right.$ $\left.\left.\left.u_{B}\right]\right\}\right)>0$. Therefore, $H(k)$ is a monotonically increasing function of $k$. According to the theory of random dominance of the network, the average degree $k_{B^{\prime}}>k_{B} \Rightarrow P_{B^{\prime}}$ is strictly first-order random dominant $P_{B} \Rightarrow \sum_{k_{B}} H\left(k_{B^{\prime}}\right) p\left(k_{B^{\prime}}\right)>$ $\sum_{k_{B}} H\left(k_{B}\right) p\left(k_{B}\right) . \quad \forall \Theta^{*}>0, \quad G\left(\Theta^{*}\right)>G\left(\Theta^{*}\right) \Rightarrow \forall \Theta_{2}^{*}>0$, and $G\left(\Theta_{2}^{*^{\prime}}\right)>G\left(\Theta_{2}^{*}\right)$. Therefore, $\Theta_{2}^{*} \geq G\left(\Theta_{2}^{*^{\prime}}\right)>G\left(\Theta_{2}^{*}\right)$. This notion contradicts $\Theta_{2}^{*}=G\left(\Theta_{2}^{*}\right)$. Therefore, the original conclusion (1) holds.

Conclusion (1) shows that when the average degree of bank credit-related network $B^{\prime}$ is greater than the average degree of bank credit-related network $B, \Theta_{2}^{*^{\prime}}>\Theta_{2}^{*} . i_{k_{B}}^{*}=$ $\eta_{B}\left[\lambda_{B} k_{B} \Theta_{2}^{*}+\lambda_{A \longrightarrow B} \lambda_{A}\left\langle k_{A}\right\rangle \Theta_{1}^{*}\right] / \eta_{B}\left[\lambda_{B} k_{B} \Theta_{2}^{*}+\lambda_{A \longrightarrow B}\right.$ $\left.\lambda_{A}\left\langle k_{A}\right\rangle \Theta_{1}^{*}\right]+\phi_{B}\left[\eta_{B}+\lambda_{B} k_{B} \Theta_{2}^{*}+\lambda_{A \longrightarrow B} \lambda_{A}\left\langle k_{A}\right\rangle \Theta_{1}^{*}+u_{B}\right]$ is a monotonically increasing function of $\Theta_{2}^{*}$. Therefore, $i_{k_{B^{\prime}}}^{*}>i_{k_{B}}^{*}$ for any $k>0$. Moreover, $\sum_{k_{B}} i_{k_{B^{\prime}}}^{*}>\sum_{k_{B}} i_{k_{B}}^{*}$; therefore, $\sum_{k_{B}}^{b} p\left(k_{B^{\prime}}\right) i_{k_{B^{\prime}}}^{*}>\sum_{k_{B}} p\left(k_{B^{\prime}}\right) i_{k_{B}}^{*}$; that is, $I_{B^{\prime}}^{*}>\sum_{k_{B}} p\left(k_{B^{\prime}}\right) i_{k_{B}}^{*} \cdot i_{k_{B}}^{*}=$ $\eta_{B}\left[\lambda_{B} k \Theta_{2}^{*}+\lambda_{A \longrightarrow B} \lambda_{A}\left\langle k_{A}\right\rangle \Theta_{1}^{*}\right] / \eta_{B}\left[\lambda_{B} k \Theta_{2}^{*}+\lambda_{A \longrightarrow B} \lambda_{A}\left\langle k_{A}\right\rangle\right.$ $\left.\Theta_{1}^{*}\right]+\phi_{B}\left[\eta_{B}+\lambda_{B} k \Theta_{2}^{*}+\lambda_{A \longrightarrow B} \lambda_{A}\left\langle k_{A}\right\rangle \Theta_{1}^{*}+u_{B}\right]$ is a monotonically increasing function of $k$. According to the theory of random dominance of the network, the average degree $k_{B^{\prime}}>$ $k_{B} \Rightarrow P_{B^{\prime}}$ is strictly a first-order random dominant $P_{B} \Rightarrow$ $\sum_{k_{B}} P\left(k_{B^{\prime}}\right) i_{k_{B^{\prime}}}^{*}>\sum_{k_{B}} P\left(k_{B}\right) i_{k_{B}}^{*}$. Therefore, $I_{B^{\prime}}^{*}>\sum_{k_{B}} P\left(k_{B^{\prime}}\right) i_{k_{B^{\prime}}}^{*}>$ $\sum_{k_{B}} P\left(k_{B}\right) i_{k_{B}}^{*}=I_{B}^{*}$. Hence, conclusion (2) was proved.

According to the infection model of Section 3.3, we can obtain $\left(\partial I_{B}^{*} / \partial \lambda_{A}\right)>0, \quad\left(\partial^{2} I_{B}^{*} / \partial \lambda_{A}^{2}\right)>0, \quad\left(\partial I_{B}^{*} / \partial \lambda_{A \rightarrow B}\right)>0$, and $\left(\partial^{2} I_{B}^{*} / \partial \lambda_{A}^{2} \longrightarrow B\right.$ ) $>0$. Therefore, the following proposition can be obtained.

Proposition 3. Bank transaction counterparty credit risk contagion scale $I_{B}^{*}$ is a monotonically increasing convex function of the credit risk contagion rate $\lambda_{A}$ of the corporate counterparty network counterparty and the risk contagion probability $\lambda_{A \longrightarrow B}$ of the corporate credit association network credit risk contagion to the bank network.

\section{Computational Simulation and Simulation Analysis of Credit Risk Contagion between the Bank and Corporate Counterparties}

4.1. Analysis of the Influencing Factors of the Credit Risk Contagion Rate Threshold of Corporate Network Counterparties. $\lambda_{A}=0.001, m=5, k=1000, u_{A}=0.01$,
$\eta_{A}=0.02$, and $\phi_{A}=0.03$ were assumed to analyze the impact of corporate counterparty heterogeneity on the credit risk contagion rate threshold $\lambda_{A}^{*}$ in corporate credit-related networks. Under the assumption of the above parameters, the characteristics of the initial economic shock $v$ and leverage level $\sigma$ of the enterprise counterparty on $i_{A}^{*}$ under $\theta=0.2, \alpha=0.3$, and $\tau=0.4$ were initially explored. Second, the characteristics of the influence $\theta$ and risk perception level $\alpha$ of the enterprise counterparty on $i_{A}^{*}$ under $\sigma=0.2, \nu=0.3$, and $\tau=0.4$ were examined. Finally, the characteristics of the influence of leverage level $\sigma$ and risk spillover ability $\tau$ of the enterprise counterparty on $i_{A}^{*}$ under $\alpha=0.2, v=0.3$, and $\theta=0.4$ were investigated.

Figure 3 depicts under three corporate credit association network structures, the influence mechanism, and evolution characteristics of the initial economic shock $\nu$, risk perception level $\alpha$, risk spillover capacity $\tau$, leverage level $\sigma$, and influence $\theta_{i}$ on the threshold of corporate counterparty credit risk contagion rate $\lambda_{A}^{*}$. Figure 3 shows that different corporate credit-related networks have different counterparty credit risk transmission rate thresholds $\lambda_{A}^{*}$. Among them, the threshold of corporate counterparty credit risk transmission rate under the BA network is the largest, followed by the exponential network and the WS smallworld network. This finding shows that different network structures significantly affect the credit risk contagion threshold $\lambda_{A}^{*}$ of corporate counterparties. This result is because of the heterogeneity of the BA network that is higher than that of the index network and the heterogeneity of the index network that is higher than that of the WS small-world network. In a network with high heterogeneity, corporate counterparties have greater resistance to the contagion of credit risk, and as the difficulty of the risk to spread increases, the threshold of counterparty credit risk contagion rate also increases.

Figure 3 verifies Proposition 1 intuitively because if the initial economic impact on the corporate counterparty is great, then the risk of contagion of the counterparty credit risk when the corporate counterparty credit risk occurs is also great. If the leverage level $\sigma$ of the corporate counterparty or the influence $\theta_{i}$ of the corporate counterparty is great, then withstanding the impact of credit risk contagion is hard when corporate counterparty credit risk occurs, resulting in faster counterparty credit risk contagion. If the risk awareness level of enterprise counterparty $\alpha$ and the risk balancing capacity of enterprise counterparty $\tau$ are great, then the enterprise's ability to deal with the credit crisis and resist the credit risk is strong, and the probability of enterprise default is small.

Tables $1-5$ profoundly reflect three different enterprise credit associations under the network structure, enterprise counterparties, the initial economic shock $v$ of corporate counterparties, the leverage level of corporate counterparties $\sigma$, the influence of corporate counterparties $\theta_{i}$, and the perception of corporate counterparty risks $\alpha$ to the enterprise credit risk contagion rate threshold value $\lambda_{A}^{*}$ impact sensitivity. The sensitivity analysis shows that the threshold $\lambda_{A}^{*}$ of corporate counterparty credit risk contagion is the monotonic decreasing function of the initial economic 


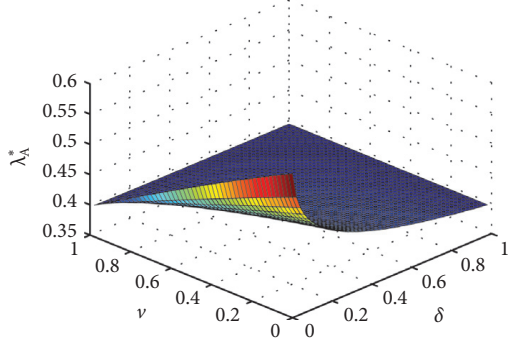

(a)

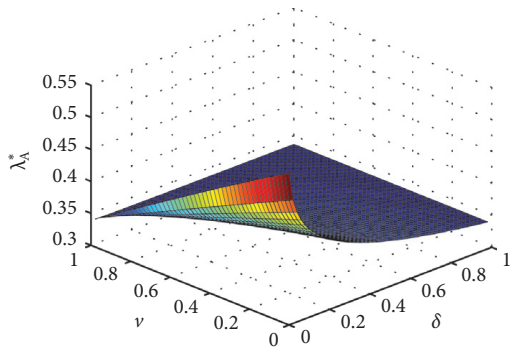

(d)

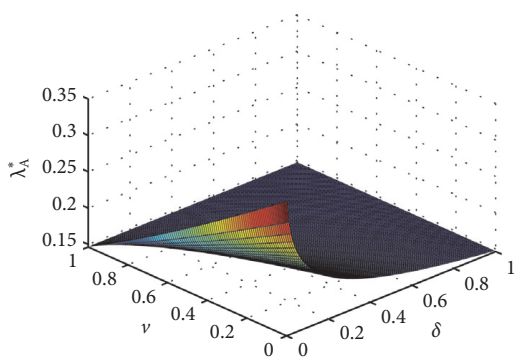

(g)

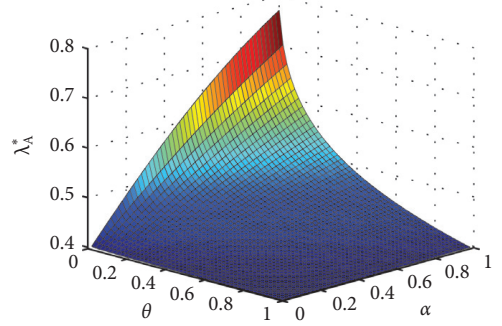

(b)

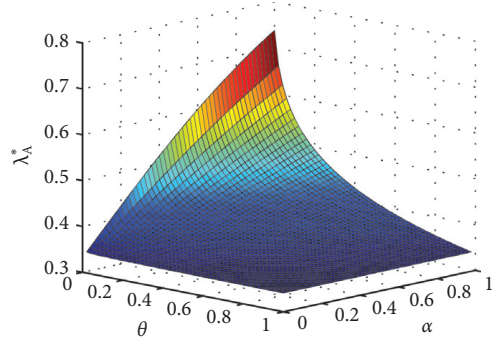

(e)

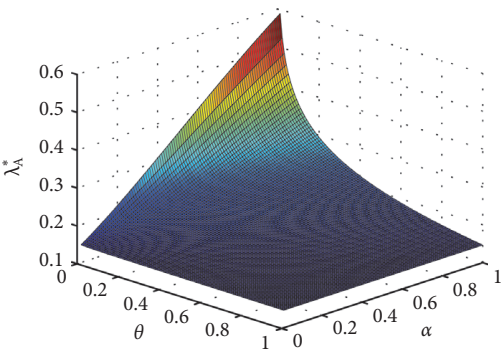

(h)

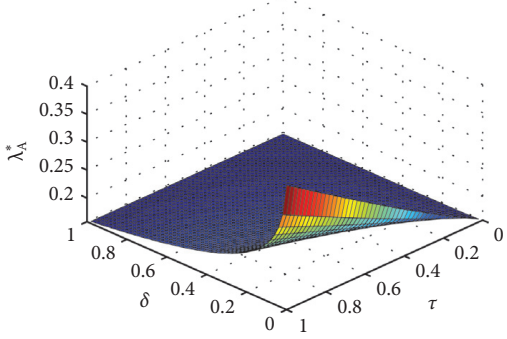

(c)

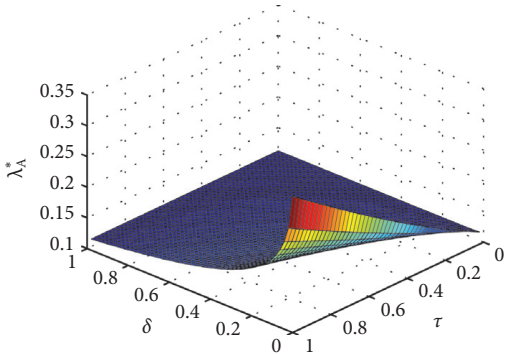

(f)

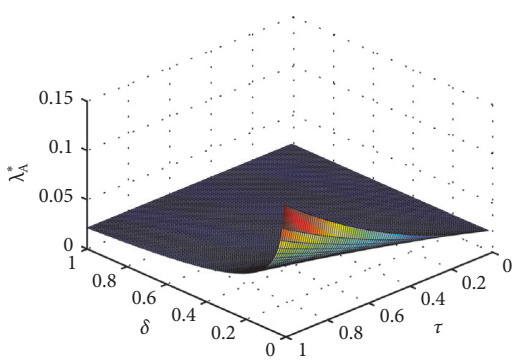

(i)

FiguRE 3: Variation law of the counterparty's credit risk contagion rate threshold under different corporate credit-related network structures. (a) The initial economic impact of the enterprise's counterparty $v$ and the impact of the enterprise's counterparty leverage level $\sigma$ on $\lambda_{A}^{*}$ under the BA network. (b) The enterprise's counterparty influence $\theta$ and the enterprise's counterparty risk perception level $\alpha$ on $\lambda_{A}^{*}$ under the BA network. (c) The leverage level $\sigma$ of the corporate counterparty and the impact of the risk-overflow ability $\tau$ of the corporate counterparty on $\lambda_{A}^{*}$ under the BA network. (d) The initial economic impact of the enterprise's counterparty $v$ and the impact of the enterprise's counterparty leverage level $\sigma$ on $\lambda_{A}^{*}$ under the index network. (e) The enterprise's counterparty influence $\theta$ and the enterprise's counterparty risk perception level $\alpha$ on $\lambda_{A}^{*}$ under the index network. (f) The leverage level $\sigma$ of the corporate counterparty and the impact of the risk-overflow ability $\tau$ of the corporate counterparty on $\lambda_{A}^{*}$ under the index network. $(\mathrm{g})$ The initial economic impact of the enterprise's counterparty $\nu$ and the impact of the enterprise's counterparty leverage level $\sigma$ on $\lambda_{A}^{*}$ under the WS small-world network (node long-distance connection probability $p=0.05$ ). (h) The enterprise's counterparty influence $\theta$ and risk perception level $\alpha$ on $\lambda_{A}^{*}$ under WS small-world network (node long-distance connection probability $p=0.05$ ). (i) The leverage level $\sigma$ of the corporate counterparty and the impact of the risk-overflow ability $\tau$ of the corporate counterparty on $\lambda_{A}^{*}$ under the WS small-world network (node long-distance connection probability $p=0.05)$.

TABLe 1: Sensitivity analysis of the influence of $\nu$ and $\sigma$ on $\lambda_{A}^{*}$ under the BA network.

\begin{tabular}{|c|c|c|c|c|c|c|c|c|c|c|c|}
\hline \multirow{2}{*}{$v$} & & \multirow{2}{*}{ Expectation } & \multirow{2}{*}{ Variance } \\
\hline & 0.1 & 0.2 & 0.3 & 0.4 & 0.5 & 0.6 & 0.7 & 0.8 & 0.9 & & \\
\hline 0.1 & 0.586078 & 0.559808 & 0.544008 & 0.532614 & 0.523677 & 0.516312 & 0.510042 & 0.504581 & 0.499741 & 0.530762 & 0.000802 \\
\hline 0.2 & 0.584346 & 0.558282 & 0.542613 & 0.531318 & 0.522458 & 0.515159 & 0.508946 & 0.503534 & 0.498738 & & 0.000789 \\
\hline 0.3 & 0.582609 & 0.556753 & 0.541216 & 0.530019 & 0.521238 & 0.514004 & 0.507848 & 0.502486 & 0.497735 & 0.528 & 0.000775 \\
\hline 0.4 & 0.580867 & 0.555221 & 0.539817 & 0.528718 & 0.520016 & 0.512849 & 0.50675 & 0.501438 & $0.496 ?$ & & 0.000762 \\
\hline 0.5 & 0.579121 & 0.553685 & 0.538415 & 0.527416 & 0.518793 & 0.511692 & 0.50565 & 0.500388 & 0.495727 & 0.525654 & 0.000748 \\
\hline 0.6 & 0.577371 & 0.552147 & 0.537012 & 0.526111 & 0.517569 & 0.510534 & 0.504549 & 0.499338 & 0.494721 & 0.524372 & 0.000735 \\
\hline 0.7 & 0.575616 & 0.550606 & 0.535606 & 0.524805 & 0.516343 & 0.509374 & 0.503447 & 0.498286 & 0.493715 & 0.523089 & 0.000722 \\
\hline 0.8 & 0.573857 & 0.549063 & 0.534197 & 0.523498 & 0.515115 & 0.508214 & 0.502344 & 0.497234 & 0.492707 & & 0.000708 \\
\hline 0.9 & 0.572093 & 0.547516 & 0.532787 & 0.522188 & 0.513886 & 0.507052 & 0.50124 & 0.49618 & 0.491699 & 0.520516 & 0.000695 \\
\hline
\end{tabular}


TABLE 2: Sensitivity analysis of the influence of $\nu$ and $\sigma$ on $\lambda_{A}^{*}$ under the index network.

\begin{tabular}{|c|c|c|c|c|c|c|c|c|c|c|c|}
\hline \multirow{2}{*}{$v$} & & \multirow{2}{*}{ Expectation } & \multirow{2}{*}{ Variance } \\
\hline & 0.1 & 0.2 & 0.3 & 0.4 & 0.5 & 0.6 & 0.7 & 0.8 & 0.9 & & \\
\hline 0.1 & 0.534871 & 0.506902 & 0.490188 & 0.478187 & 0.468803 & 0.461091 & 0.45454 & 0.448846 & 0.443808 & 0.47636 & 0.000892 \\
\hline 0.2 & 0.53302 & 0.505284 & 0.488717 & 0.476824 & 0.467526 & 0.459885 & 0.453396 & 0.447756 & 0.442766 & 0.475019 & 0.000876 \\
\hline 0.3 & 0.531164 & 0.503664 & 0.487244 & 0.475459 & 0.466247 & 0.458679 & 0.452252 & 0.446665 & 0.441723 & 0.473677 & 0.00086 \\
\hline 0.4 & 0.529305 & 0.502041 & 0.485769 & 0.474093 & 0.464968 & 0.457471 & 0.451106 & 0.445573 & 0.44068 & 0.472334 & 0.000844 \\
\hline 0.5 & 0.527443 & 0.500416 & 0.484292 & 0.472725 & 0.463687 & 0.456263 & 0.44996 & 0.444481 & 0.439636 & 0.470989 & 0.000829 \\
\hline 0.6 & 0.525576 & 0.498788 & 0.482813 & 0.471357 & 0.462406 & 0.455054 & 0.448813 & 0.443389 & 0.438592 & 0.469643 & 0.000813 \\
\hline 0.7 & 0.523706 & 0.497159 & 0.481333 & 0.469986 & 0.461123 & 0.453844 & 0.447665 & 0.442296 & 0.437547 & 0.468295 & 0.000798 \\
\hline 0.8 & 0.521832 & 0.495526 & 0.479852 & 0.468615 & 0.459839 & 0.452633 & 0.446516 & 0.441202 & 0.436502 & 0.466946 & 0.000783 \\
\hline 0.9 & 0.519955 & 0.493892 & 0.478368 & 0.467242 & 0.458555 & 0.451421 & 0.445367 & 0.440107 & 0.435456 & 0.465596 & 0.000767 \\
\hline
\end{tabular}

TABLE 3: Sensitivity analysis of the influence of $\nu$ and $\sigma$ on $\lambda_{A}^{*}$ under the WS small-world network.

\begin{tabular}{|c|c|c|c|c|c|c|c|c|c|c|c|}
\hline \multirow{2}{*}{$v$} & \multicolumn{9}{|c|}{$\sigma$} & \multirow{2}{*}{ Expectation } & \multirow{2}{*}{ Variance } \\
\hline & 0.1 & 0.2 & 0.3 & 0.4 & 0.5 & 0.6 & 0.7 & 0.8 & 0.9 & & \\
\hline 0.1 & 0.329092 & 0.29915 & 0.281854 & 0.269714 & 0.260385 & 0.252825 & 0.246481 & 0.241022 & 0.236238 & 0.268529 & 0.000923 \\
\hline 0.2 & 0.327072 & 0.297456 & 0.280353 & 0.26835 & 0.259126 & 0.251652 & 0.24538 & 0.239984 & 0.235254 & 0.267181 & 0.000902 \\
\hline 0.3 & 0.325053 & 0.295764 & 0.278854 & 0.266987 & 0.257869 & 0.25048 & 0.24428 & 0.238946 & 0.234271 & 0.265834 & 0.000882 \\
\hline 0.4 & 0.323035 & 0.294073 & 0.277356 & 0.265626 & 0.256613 & 0.24931 & 0.243182 & 0.23791 & 0.233289 & 0.264488 & 0.000862 \\
\hline 0.5 & 0.321018 & 0.292384 & 0.27586 & 0.264267 & 0.255359 & 0.248142 & 0.242086 & 0.236875 & 0.232308 & 0.263144 & 0.000842 \\
\hline 0.6 & 0.319003 & 0.290697 & 0.274366 & 0.262909 & 0.254107 & 0.246975 & 0.240991 & 0.235842 & 0.231329 & 0.261802 & 0.000823 \\
\hline 0.7 & 0.31699 & 0.289012 & 0.272874 & 0.261553 & 0.252856 & 0.24581 & 0.239897 & 0.23481 & 0.230351 & 0.260462 & 0.000803 \\
\hline 0.8 & 0.314978 & 0.287329 & 0.271384 & 0.260199 & 0.251607 & 0.244646 & 0.238805 & 0.23378 & 0.229375 & 0.259123 & 0.000784 \\
\hline 0.9 & 0.312968 & 0.285648 & 0.269896 & 0.258847 & 0.25036 & 0.243484 & 0.237714 & 0.232751 & 0.2284 & 0.257785 & 0.000765 \\
\hline
\end{tabular}

Table 4: Sensitivity analysis of the impact of $\tau$ and $\sigma$ on $\lambda_{A}^{*}$ under the BA network.

\begin{tabular}{|c|c|c|c|c|c|c|c|c|c|c|c|}
\hline \multirow{2}{*}{$\tau$} & \multicolumn{9}{|c|}{$\sigma$} & \multirow{2}{*}{ Expectation } & \multirow{2}{*}{ Variance } \\
\hline & 0.1 & 0.2 & 0.3 & 0.4 & 0.5 & 0.6 & 0.7 & 0.8 & 0.9 & & \\
\hline 0.1 & 0.159627 & 0.159343 & 0.159176 & 0.159059 & 0.158967 & 0.158893 & 0.15883 & 0.158775 & 0.158727 & $1.59 E-01$ & $8.68 E-08$ \\
\hline 0.2 & 0.16152 & 0.160949 & 0.160615 & 0.160379 & 0.160196 & 0.160046 & 0.159919 & 0.15981 & 0.1597 & $1.60 E-01$ & $3.50 E-07$ \\
\hline 0.3 & 0.163424 & 0.162563 & 0.16206 & 0.161704 & 0.161428 & 0.161203 & 0.161012 & 0.160848 & 0.160 & $1.62 E-01$ & $7.93 E-07$ \\
\hline 0.4 & 0.165337 & 0.164184 & 163511 & 0.163034 & 0.162665 & 0.162364 & 0.162109 & 0.161888 & 0.161694 & $1.63 E-01$ & $1.42 E-06$ \\
\hline 0.5 & 0.167261 & 0.165812 & 0.164968 & 0.164369 & 0.163906 & 0.163528 & 0.163209 & 0.162932 & 0.162689 & & $2.24 E-06$ \\
\hline 0.6 & 0.169194 & 0.167448 & 0.16643 & 0.165709 & 0.165151 & 0.164696 & 0.164312 & 0.163979 & 0.163686 & $1.66 E-01$ & $3.25 E-06$ \\
\hline 0.7 & 0.171137 & 0.16909 & 0.167898 & 0.167054 & 0.166401 & 0.165868 & 0.165418 & 0.165029 & 0.164686 & $1.67 E-01$ & $4.46 E-06$ \\
\hline 0.8 & 0.173089 & 0.170739 & 0.169371 & 0.168404 & 0.167655 & 0.167044 & 0.166528 & 0.166082 & 0.165689 & $1.68 E-01$ & $5.87 E-06$ \\
\hline 0.9 & 0.175051 & 0.172396 & 0.170851 & 0.169758 & 0.168912 & 0.168223 & 0.167641 & 0.167137 & 0.166694 & $1.70 E-01$ & $7.48 E-06$ \\
\hline
\end{tabular}

TABLe 5: Sensitivity analysis of the impact of $\theta$ and $\alpha$ on $\lambda_{A}^{*}$ under the BA network.

\begin{tabular}{|c|c|c|c|c|c|c|c|c|c|c|c|}
\hline \multirow{2}{*}{$\alpha$} & \multicolumn{9}{|c|}{$\theta$} & \multirow[b]{2}{*}{ Expectation } & \multirow[b]{2}{*}{ Variance } \\
\hline & 0.1 & 0.2 & 0.3 & 0.4 & 0.5 & 0.6 & 0.7 & 0.8 & 0.9 & & \\
\hline 0.1 & 0.401896 & 0.401185 & 0.400769 & 0.400473 & 0.400244 & 0.400057 & 0.399899 & 0.399762 & 0.399641 & $4.00 E-01$ & $5.45 E-07$ \\
\hline 0.2 & 0.406617 & 0.405196 & 0.404365 & 0.403775 & 0.403318 & 0.402944 & 0.402627 & 0.402353 & 0.402112 & $4.04 E-01$ & $2.17 E-06$ \\
\hline 0.3 & 0.411332 & 0.409204 & 0.407958 & 0.407074 & 0.406388 & 0.405828 & 0.405354 & 0.404943 & 0.404581 & $4.07 E-01$ & $4.88 E-06$ \\
\hline 0.4 & 0.41604 & 0.413206 & 0.411547 & 0.41037 & 0.409456 & 0.40871 & 0.408078 & 0.407531 & 0.407049 & $4.10 E-01$ & $8.66 E-06$ \\
\hline 0.5 & 0.420741 & 0.417204 & 0.415133 & 0.413663 & 0.412522 & 0.411589 & 0.410801 & 0.410117 & 0.409514 & $4.13 E-01$ & $1.35 E-05$ \\
\hline 0.6 & 0.425434 & 0.421196 & 0.418714 & 0.416952 & 0.415584 & 0.414466 & 0.413521 & 0.412701 & 0.411979 & $4.17 E-01$ & $1.94 E-05$ \\
\hline 0.7 & 0.430117 & 0.425182 & 0.422291 & 0.420237 & 0.418643 & 0.417341 & 0.416238 & 0.415283 & 0.414441 & $4.20 E-01$ & $2.63 E-05$ \\
\hline 0.8 & 0.434792 & 0.429162 & 0.425863 & 0.423519 & 0.421699 & 0.420212 & 0.418954 & 0.417863 & 0.416901 & $4.23 E-01$ & $3.43 E-05$ \\
\hline 0.9 & 0.439456 & 0.433135 & 0.429429 & 0.426796 & 0.424752 & 0.42308 & 0.421666 & 0.420441 & 0.419359 & $4.26 E-01$ & $4.33 E-05$ \\
\hline
\end{tabular}

impact of the corporate counterparty $\nu$, the leverage level of the corporate counterparty $\sigma$, and the influence of the corporate counterparty $\theta_{i}$, with the decreasing marginal. The threshold $\lambda_{A}^{*}$ of corporate counterparty credit risk contagion is a monotonically increasing function of the risk perception level $\alpha$ and the risk leveling capacity $\tau$ of the corporate counterparty, with a diminishing margin. Moreover, Tables 1-5 further confirm Proposition 1. 
4.2. Analysis of Influencing Factors of the Scale of Credit Risk Contagion of Corporate Network Counterparties. $\lambda_{A}=0.001, m=5, k=1000, u_{A}=0.01, \eta_{A}=0.02$, and $\phi_{A}=$ 0.03 were assumed to analyze the impact of corporate counterparty heterogeneity on corporate counterparty credit risk contagion scale $i_{A}^{*}$. Under the abovementioned parameter assumptions, the characteristics of the initial economic shock $v$ and leverage level $\sigma$ of the enterprise counterparty on $i_{A}^{*}$ under $\theta=\alpha=\tau=0.2$ were initially explored. Second, the characteristics of the influence of the enterprise counterparty influence $\theta$ and risk perception level $\alpha$ on $i_{A}^{*}$ under $\sigma=\nu=\tau=0.2$ were examined. Last, the characteristics of the influence of the leverage level $\sigma$ and risk-overflow ability $\tau$ of the enterprise counterparty on $i_{A}^{*}$ under $\alpha=\nu=\theta=0.2$ were analyzed.

Figure 4 depicts the influence mechanism and evolution characteristics of the initial economic shock $\nu$, risk perception level $\alpha$, risk spillover ability $\tau$, leverage level $\sigma$, and influence $\theta_{i}$ of corporate counterparties on the credit risk contagion scale $i_{A}^{*}$ of corporate counterparties under different enterprise credit association network structures. Figures 4(a)-4(c) show that if the heterogeneity of an enterprise's credit-related network is high, then the corresponding counterparty credit risk contagion scale $i_{A}^{*}$ is small. This finding is because the direct edges of corporate counterparties in the BA network are lower than those in the index network. On the contrary, the direct edges of corporate counterparties in the index network are lower than those in the WS small-world network, and if many direct edges in the network exist, then the degree of business correlation between counterparties is high and passing risks to related counterparties is easy when a credit crisis occurs. Figure 4(a) shows that the increase in the initial economic shock $v$ of the corporate counterparty will increase the scale $i_{A}^{*}$ of the corporate counterparty credit risk contagion. That is, the initial economic shock $v$ of the corporate counterparty will promote the credit risk contagion of the enterprise's counterparty, and the marginal increase will occur. In addition, Figure 4(a) depicts that as the leverage level $\sigma$ of corporate counterparties increases, the scale $i_{A}^{*}$ of corporate counterparty credit risk contagion continues to increase. Figures 4(b) and 4(c) show that the increase in the enterprise transaction counterparty's risk perception level $\alpha$ and the enterprise transaction counterparty's risk-overflow capacity $\tau$ will lead to a reduction in the enterprise transaction counterparty's credit risk transmission scale $i_{A}^{*}$. In other words, the increase in the counterparty risk perception level $\alpha$ and the risk balance ability of the corporate counterparty $\tau$ can inhibit the credit risk contagion of enterprise counterparty. The counterparty influence $\theta_{i}$ of the corporate counterparty promotes the scale of the corporate counterparty credit risk contagion $i_{A}^{*}$.

$\lambda_{A}=0.001, m=5, k_{A}=1000, u_{A}=0.01, \eta_{A}=0.02$, and $\phi_{A}=0.03$ were assumed to analyze the influence mechanism of the degree $k$ of the enterprise's credit-related network on the scale $i_{A}^{*}$ of the enterprise's counterparty credit risk. Under the assumption of the above parameters, we initially explore the mechanism and evolution characteristics of the degree $k$ of the enterprise credit-related network on $i_{A}^{*}$ under the condition of $\theta=\alpha=\tau=\sigma=0.2$ and the initial economic shock $v$ of different network structures and business counterparties. Second, the mechanism and evolution characteristics of the degree $k$ of the enterprise credit-related network on $i_{A}^{*}$ under the condition of $\theta=\nu=\tau=\sigma=0.2$ and different network structures and enterprise transaction counterparty risk perception levels $\alpha$ were explored. Third, the influence mechanism and evolution characteristics of the degree $k$ of the enterprise's credit-related network on $i_{A}^{*}$ under the condition of $\alpha=\nu=\sigma=\tau=0.4$ and different network structures and the influence of different business counterparties $\theta_{i}$ were investigated. Fourth, the influence mechanism and evolution characteristics of the degree $k$ of the enterprise's credit-related network on $i_{A}^{*}$ under the condition of $\sigma=v=\theta=\alpha=0.4$ and different network structures and the risk-overflow capacity $\tau$ of different enterprise counterparties were examined. Finally, the influence mechanism and evolution characteristics of the degree $k$ of the enterprise's credit-related network on $i_{A}^{*}$ under the condition of $\alpha=\nu=\theta=\tau=0.2$ and different network structures and the leverage level $\sigma$ of different enterprise counterparties were analyzed.

Figure 5 shows the relationship between the credit risk contagion scale $i_{A}^{*}$ of corporate counterparty and the degree $k$ of corporate counterparty under different corporate credit association networks. In addition, the scale of corporate counterparty credit risk contagion $i_{A}^{*}$ under the WS smallworld network is larger than that under the index network. By contrast, the scale of corporate counterparty credit risk contagion $i_{A}^{*}$ under the index network is larger than that under the BA network. This result is because the heterogeneity of the BA network is higher than that of the index network, and the heterogeneity of the index network is higher than that of the WS small-world network. If the heterogeneity of corporate counterparty in the network is high, then the resistance of counterparty credit risk contagion is great. If the risk diffusion is difficult, then the scale of corporate counterparty credit risk contagion is low. Figure 5 shows that the credit risk contagion scale $i_{A}^{*}$ of corporate counterparty under different corporate credit association network structures tends to a stable value with the increase in the degree $k$ of the corporate counterparty.

4.3. Analysis of the Influencing Factors of the Threshold of the Credit Risk Contagion Rate of the Bank's Network Counterparty. $\lambda_{A}=0.1,\left\langle k_{A}\right\rangle=9, \Theta_{1}^{*}=0.5, \lambda_{A \longrightarrow B}=0.09$, $m=5, k_{B}=1000, u_{A}=\eta_{A}=\phi_{A}=0.2$, and $\eta_{B}=u_{B}=\phi_{B}=$ 0.2 were assumed to analyze the impact of bank transaction counterparty heterogeneity on the bank network credit risk contagion rate threshold $\lambda_{B}^{*}$. Under the assumptions of the above parameters, the influence of the degree of business association of the bank's counterparty $\varepsilon$ and its risk resistance $\omega$ on $\lambda_{B}^{*}$ under $\rho=0.2$ was initially explored. Second, the influence of the bank's counterparty risk resistance ability $\omega$ and risk information processing ability $\rho$ on $\lambda_{B}^{*}$ under $\varepsilon=0.4$ was investigated. Last, the influence of the processing capacity $\rho$ of bank counterparty risk information and the degree of business relevance $\varepsilon$ on $\lambda_{B}^{*}$ under $\omega=0.3$ was examined. 


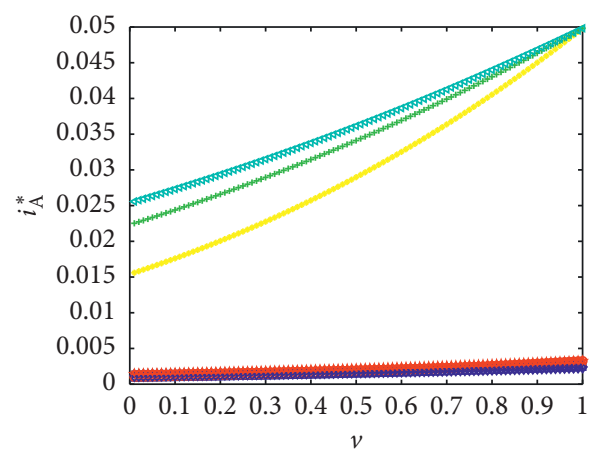

- The BA network, $\sigma=0.2$

The WS small-world network, $\sigma=0.2$

- The index network, $\sigma=0.2$

- The BA network, $\sigma=0.5$

* The WS small-world network, $\sigma=0.5$

* The index network, $\sigma=0.5$

$\checkmark$ The BA network, $\sigma=0.7$

$\triangleleft$ The WS small-world network, $\sigma=0.7$

* The index network, $\sigma=0.7$

(a)

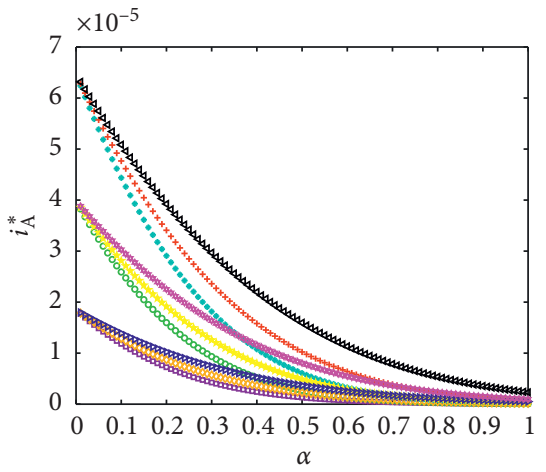

- The BA network, $\theta=0.3$

- The WS small-world network, $\theta=0.3$

- The index network, $\theta=0.3$

The BA network, $\theta=0.5$

+ The WS small-world network, $\theta=0.5$

The index network, $\theta=0.5$

- The BA network, $\theta=0.8$

$\triangleleft$ The WS small-world network, $\theta=0.8$

* The index network, $\theta=0.8$

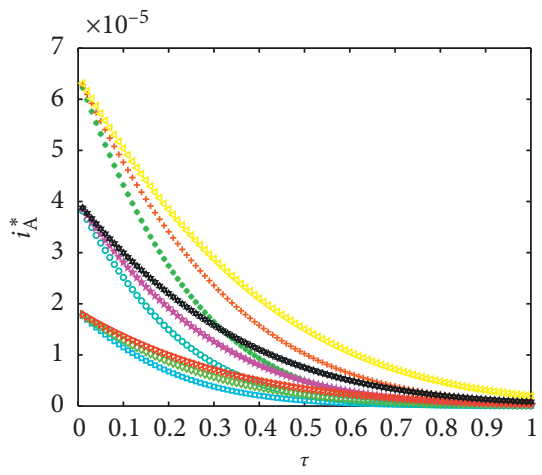

- The BA network, $\sigma=0.25$

- The WS small-world network, $\sigma=0.25$

- The index network, $\sigma=0.25$

- The BA network, $\sigma=0.5$

+ The WS small-world network, $\sigma=0.5$

* The index network, $\sigma=0.5$

$>$ The BA network, $\sigma=0.75$

The WS small-world network, $\sigma=0.75$

* The index network, $\sigma=0.75$

(b)

(c)

FIGURE 4: Evolutionary law of the scale of credit risk contagion of corporate counterparties. (a) The impact of the initial economic shock $v$ of the corporate counterparty on $i_{A}^{*}$ under the different network structures and the leverage level $\sigma$ of different corporate counterparties. (b) The impact of the enterprise transaction counterparty's risk perception level $\alpha$ on $i_{A}^{*}$ under the different network structures and the influence of different corporate counterparties $\theta$. (c) The impact of the risk-overflow ability $\tau$ of corporate counterparties on $i_{A}^{*}$ under different network structures and the leverage level $\sigma$ of different corporate counterparties.

Figure 6 depicts the mechanism and evolution characteristics of the bank's counterparty business relationship $\varepsilon$, risk resistance $\rho$, and risk information processing ability $\lambda_{B}^{*}$ on the bank's counterparty credit risk contagion rate threshold $\lambda_{B}^{*}$ under two network structures. Figure 6 shows that different banking networks have different thresholds $\lambda_{B}^{*}$ for the spread of counterparty credit risk. Among them, the threshold of the credit risk contagion rate of bank counterparties under the index network is the largest than the threshold $\lambda_{B}^{*}$ of the credit risk contagion rate of bank counterparties under the WS small-world network. This finding shows that different credit network structures significantly affect the credit risk threshold of bank counterparties because the heterogeneity of the index network is higher than that of the WS small-world network. If the heterogeneity of the bank's counterparty in the network is high, then the resistance of the counterparty's credit risk contagion is great. If spreading the risk is difficult, then the threshold of the counterparty's credit risk contagion is high. Figure 6 also verifies Inference 2 intuitively.

Tables 6-9 show the bank credit association network under two different network structures, the influence sensitivity of counterparty business association degree $\varepsilon$, risk resistance ability $\omega$, and risk information processing ability $\rho$ on the bank counterparty credit risk contagion threshold $\lambda_{B}^{*}$. Sensitivity analysis shows that the credit risk contagion threshold $\lambda_{B}^{*}$ of the bank counterparty is a monotonously decreasing function of the bank's counterparty business relevance $\varepsilon$, a monotonically increasing function of the bank's counterparty risk resistance $\omega$, and the bank's counterparty risk information processing ability $\rho$. Similarly, the degree of business association of a bank's counterparty $\varepsilon$ decreases with a marginal increase to the credit risk contagion threshold of the bank's counterparty $\lambda_{B}^{*}$. The bank counterparty risk resistance $\omega$ has an increasing feature of a marginal decrease to the threshold value of counterparty credit risk contagion rate $\lambda_{B}^{*}$. The processing capacity of bank counterparty risk information $\rho$ has an increasing feature of a marginal decrease to the threshold value of counterparty credit risk contagion rate $\lambda_{B}^{*}$.

4.4. Analysis of the Influencing Factors of the Credit Risk Contagion Scale of the Bank's Online Counterparty. $\lambda_{A}=0.1, \lambda_{B}=0.001,\left\langle k_{A}\right\rangle=9, \Theta_{1}^{*}=0.5, \lambda_{A \longrightarrow B}=0.09$, $m=5, k_{B}=1000, \mathrm{u}_{A}=\eta_{A}=\phi_{A}=0.2$, and $\eta_{B}=u_{B}=\phi_{B}=$ 0.2 were assumed to analyze the impact mechanism of bank counterparty heterogeneity on bank counterparty credit risk contagion scale $i_{B}^{*}$. Under the assumption of the above parameters, the influence of the degree of the business relevance of the bank's counterparty $\varepsilon$ and the ability of risk resistance $\omega$ on $i_{B}^{*}$ under $\rho=0.2$ was initially explored. Second, the influence of the bank's counterparty risk resistance ability $\omega$ and risk information processing ability $\rho$ on $i_{B}^{*}$ under $\varepsilon=0.4$ was investigated. Last, the influence of bank transaction counterparts' business relevance $\varepsilon$ and risk resistance $\omega$ on $i_{B}^{*}$ under $\rho=0.5$ was examined.

Figure 7 depicts the bank's credit-related network under different network structures, the influence mechanism, and evolution characteristics of the bank's counterparty business connection degree $\varepsilon$, risk information processing ability $\rho$, 


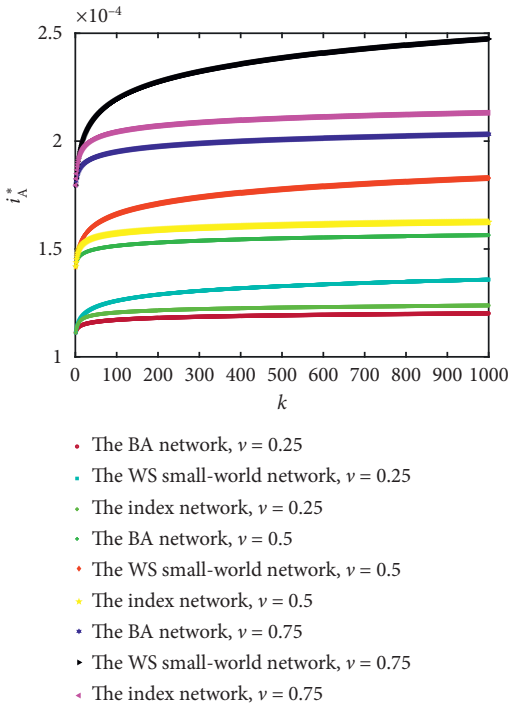

(a)

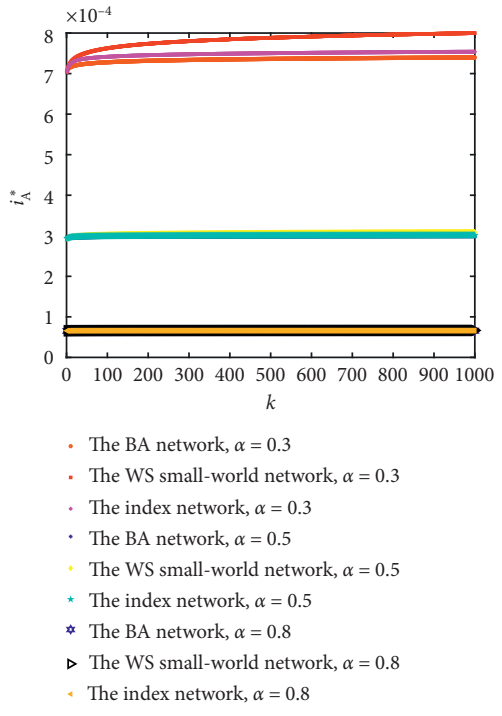

(b)

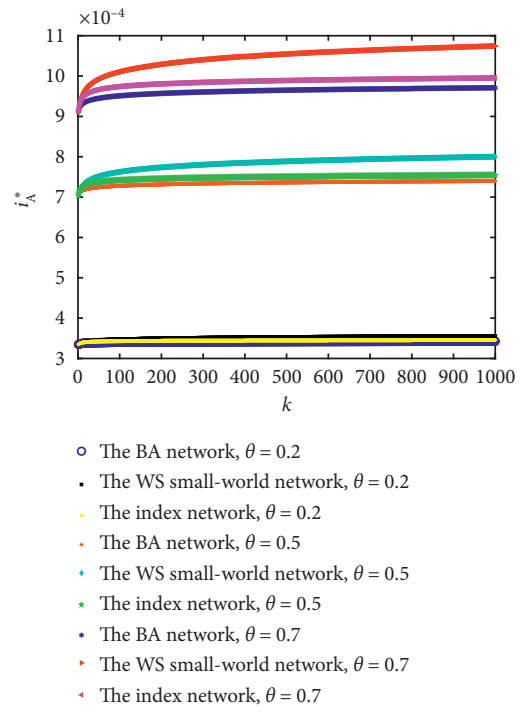

(c)

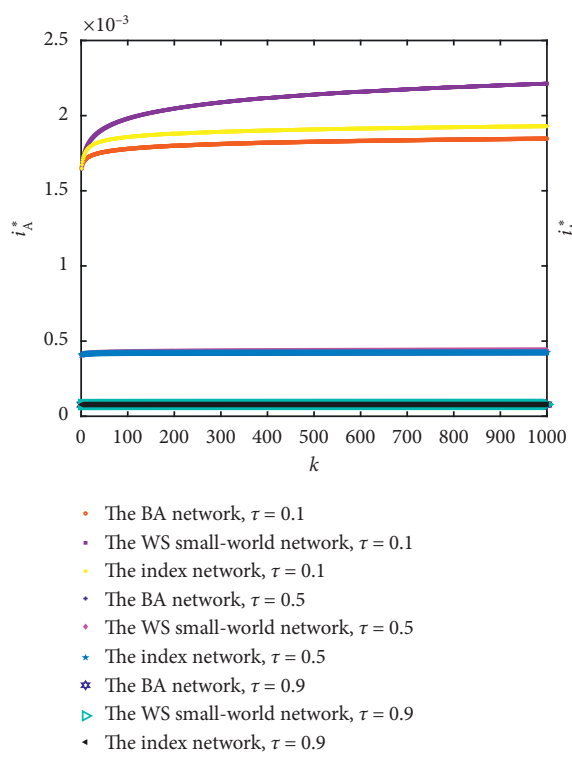

(d)

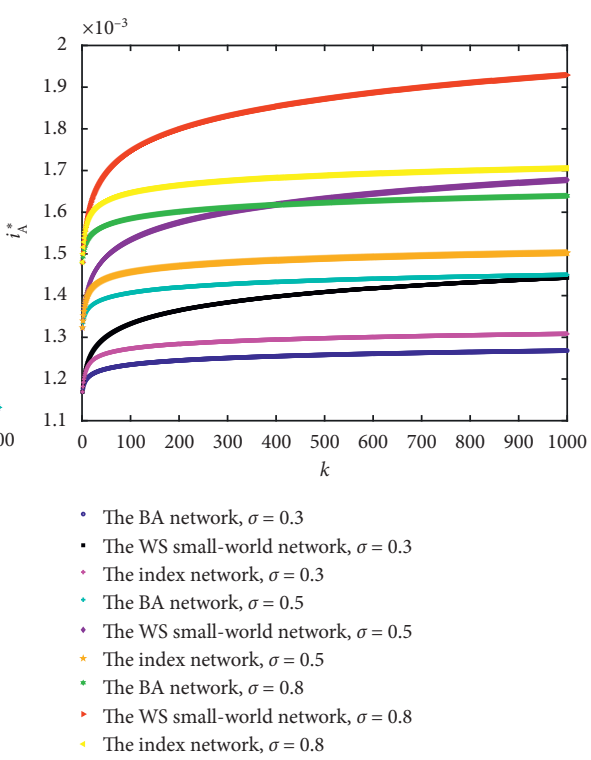

(e)

Figure 5: Evolutionary characteristics of the impact of the degree of business counterparties. (a) The impact of the enterprise counterparty degree $k$ on $i_{A}^{*}$ under different network structures and business counterparty initial economic shocks $\nu$. (b) The impact of the enterprise counterparty degree $k$ on $i_{A}^{*}$ under different network structures and different enterprise counterparty risk perception levels $\alpha$. (c) The impact of the enterprise counterparty degree $k$ on $i_{A}^{*}$ under different network structures and influence $\theta_{i}$ of the enterprise counterparty. (d) The impact of the corporate counterparty's degree $k$ on $i_{A}^{*}$ under different network structures and different the risk spillover abilities of corporate counterparties $\tau$. (e) The impact of the enterprise counterparty degree $k$ on $i_{A}^{*}$ under different network structures and different corporate counterparties' leverage levels $\sigma$.

and risk resistance ability $\omega$ on the bank counterparty's credit risk contagion scale $i_{B}^{*}$. Figures 7(a)-7(c) show that if the heterogeneity of the banking network is high, then the scale $i_{B}^{*}$ of the corresponding counterparty credit risk contagion is small. This result is because the heterogeneity of bank counterparties in the BA network is higher than that of the index network, and the heterogeneity of the index network is higher than that of the WS network. When a credit crisis occurs, if the bank's counterparties in the network are heterogeneous, then the resistance to the spread of counterparties' credit risks is great and the probability of risk contagion to other counterparties is small. Figure 7(a) shows that the increase in the degree of bank transaction counterparty business association $\varepsilon$ will increase the scale of bank transaction counterparty credit risk contagion $i_{B}^{*}$. That is, the degree of bank transaction counterparty business association degree $\varepsilon$ will promote the credit risk contagion of a bank's counterparty, with diminishing margins. In 


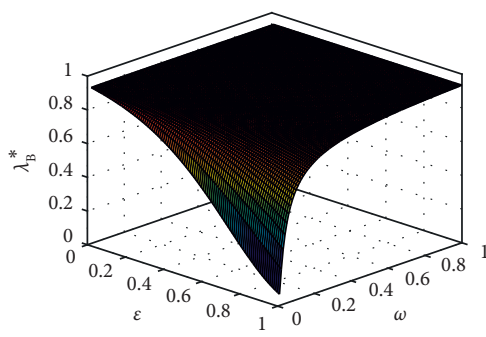

(a)

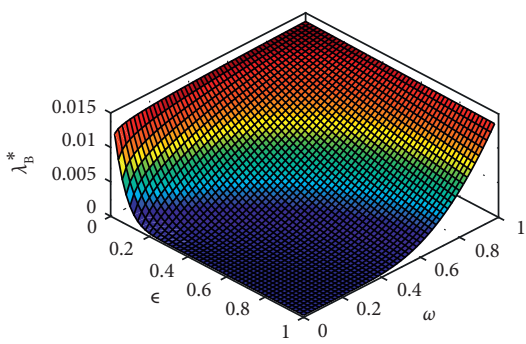

(d)

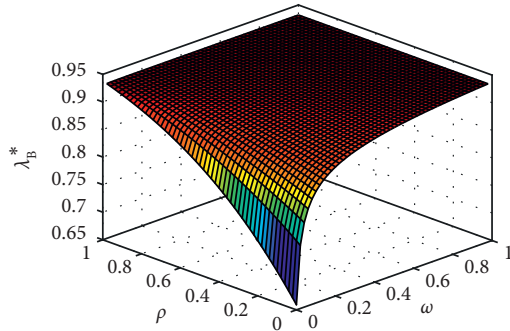

(b)

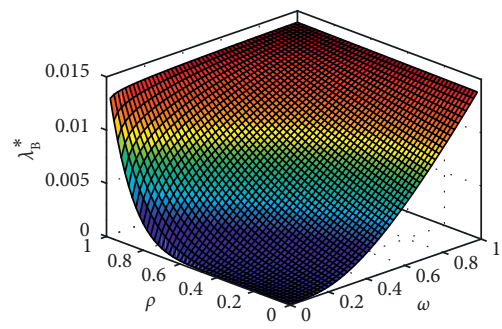

(e)

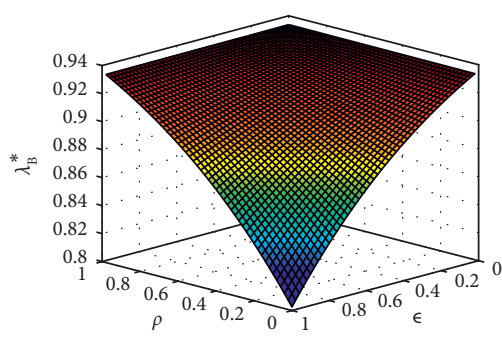

(c)

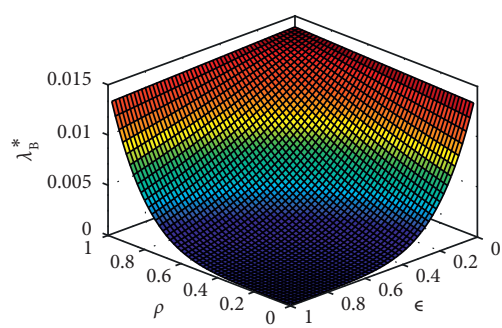

(f)

FiguRe 6: Bank credit-related network under the two network structures and the influence rule of the threshold $\lambda_{B}^{*}$ of the credit risk transmission rate of the counterparty of the bank network. (a) The influence of bank counterparty business association degree $\varepsilon$ and bank counterparty risk resistance ability $\omega$ on $\lambda_{B}^{*}$ in the bank network under the index network. (b) The influence of the bank's counterparty risk information processing $\rho$ and resistance $\omega$ abilities on $\lambda_{B}^{*}$ in the bank network under the index network. (c) The influence of the processing ability of the bank counterparty risk information $\rho$ and the degree of the bank counterparty's business relevance $\varepsilon$ on $\lambda_{B}^{*}$ in the bank network under the index network. (d) The influence of the bank transaction counterparty business association degree $\varepsilon$ and bank transaction counterparty risk resistance ability $\omega$ on $\lambda_{B}^{*}$ in the bank network under the WS network (node long-distance connection probability $p=0.05$ ). (e) The influence of the processing ability of bank counterparty risk information $\rho$ and bank counterparty risk resistance capacity $\omega$ on $\lambda_{B}^{*}$ in the bank network under the WS network (node long-distance connection probability $p=0.05$ ). (f) The influence of the bank counterparty ability to process risk information $\rho$ and the degree of the bank counterparty's business relevance $\varepsilon$ on $\lambda_{B}^{*}$ in the bank network under the WS network (node long-distance connection probability $p=0.05$ ).

TABLE 6: Bank network under the index network-the sensitivity analysis of the influence of $\varepsilon$ and $\omega$ on $\lambda_{B}^{*}$.

\begin{tabular}{|c|c|c|c|c|c|c|c|c|c|c|c|}
\hline \multirow{2}{*}{$\omega$} & & \multirow{2}{*}{ Expectation } & \multirow{2}{*}{ Variance } \\
\hline & 0.1 & 0.2 & 0.3 & 0.4 & 0.5 & 0.6 & 0.7 & 0.8 & 0.9 & & \\
\hline 0.1 & 0.932011 & 0.929551 & 0.927006 & 0.924373 & 0.921649 & 0.918831 & 0.915917 & 0.912903 & 0.909786 & 0.921337 & 5.788 \\
\hline 0.2 & 0.932374 & 0.9303 & 0.928166 & 0.925969 & 0.923708 & 0.92138 & 0.918985 & & .913984 & & \\
\hline 0.3 & 0.932585 & 0.930735 & 0.928836 & 0.926888 & 0.924888 & 0.922836 & 0.92073 & 0.918569 & 0.916352 & 0.924 & $7 E-05$ \\
\hline 0.4 & 0.932735 & 0.931042 & 0.929308 & 0.927533 & 0.925715 & 0.923852 & 0.921946 & 0.919993 & 0.917994 & 0.925569 & $2.5463 E-05$ \\
\hline 0.5 & 0.932851 & 0.931279 & 0.929672 & 0.928029 & 0.92635 & 0.924632 & 0.922877 & 0.921082 & 0.919247 & 0.926224 & $2.16867 E-05$ \\
\hline 0.6 & 0.932945 & 0.931472 & 0.929968 & 0.928433 & 0.926865 & 0.925264 & 0.923629 & 0.921961 & 0.920257 & 0.926755 & $1.88661 E-05$ \\
\hline 0.7 & 0.933025 & 0.931635 & 0.930218 & 0.928772 & 0.927298 & 0.925794 & 0.92426 & 0.922696 & 0.921101 & 0.9272 & $1.66606 E-05$ \\
\hline 0.8 & 0.933094 & 0.931776 & 0.930433 & 0.929065 & 0.927671 & 0.92625 & 0.924803 & 0.923328 & 0.921826 & 0.927583 & $1.4879 E-05$ \\
\hline 0.9 & 0.933155 & 0.9319 & 0.930623 & 0.929322 & 0.927998 & 0.92665 & 0.925278 & 0.923882 & 0.92246 & 0.927919 & $1.34042 E-05$ \\
\hline
\end{tabular}

TABLE 7: Bank network under the WS network-the sensitivity analysis of the influence of $\varepsilon$ and $\omega$ on $\lambda_{B}^{*}$.

\begin{tabular}{|c|c|c|c|c|c|c|c|c|c|c|c|}
\hline \multirow{2}{*}{$\omega$} & & \multirow{2}{*}{ Expectation } & \multirow{2}{*}{ Variance } \\
\hline & 0.1 & 0.2 & 0.3 & 0.4 & 0.5 & 0.6 & 0.7 & 0.8 & 0.9 & & \\
\hline 0.1 & 0.011977 & 0.008539 & 0.005932 & 0.004008 & 0.002628 & 0.001669 & 0.001023 & 0.000604 & 0.000343 & 0.00408 & $1.61234 E-05$ \\
\hline 0.2 & 0.01245 & 0.009666 & .007395 & 0.005572 & 0.00413 & 0.003 & 0.002 & 0.00151 & 0.001 & & \\
\hline 0.3 & 0.012674 & 0.010228 & 0.008167 & 0.00645 & 0.005035 & 0.003883 & 0.002957 & 0.002221 & 0.001646 & 0.005918 & $1.43962 E-05$ \\
\hline 0.4 & 0.012824 & 0.010612 & 0.008709 & 0.007086 & 0.005715 & 0.004566 & 0.003612 & 0.002829 & 0.002192 & 0.00646 & $1.33552 E-05$ \\
\hline 0.5 & 0.012937 & 0.010906 & 0.009132 & 0.007594 & 0.006269 & 0.005137 & 0.004176 & 0.003368 & 0.002693 & 0.006912 & $1.23818 E-05$ \\
\hline 0.6 & 0.013027 & 0.011145 & 0.009482 & 0.00802 & 0.006742 & 0.005633 & 0.004676 & 0.003856 & & & $1.14815 E-05$ \\
\hline 0.7 & 0.013103 & 0.011347 & 0.00978 & 0.008388 & 0.007158 & 0.006076 & 0.005129 & 0.004306 & 0.003594 & 0.007653 & $1.065 E-05$ \\
\hline 0.8 & 0.013168 & 0.011523 & 0.010042 & 0.008714 & 0.007529 & 0.006476 & 0.005545 & 0.004724 & 0.004006 & 0.00797 & $9.88128 E-06$ \\
\hline 0.9 & 0.013225 & 0.011678 & 0.010276 & 0.009008 & 0.007867 & 0.006844 & 0.00593 & 0.005117 & 0.004397 & 0.00826 & $9.16916 E-06$ \\
\hline
\end{tabular}


TABLE 8: Bank network under the index network-the sensitivity analysis of the influence of $\rho$ and $\omega$ on $\lambda_{B}^{*}$.

\begin{tabular}{|c|c|c|c|c|c|c|c|c|c|c|c|}
\hline \multirow{2}{*}{$\rho$} & \multicolumn{9}{|c|}{$\omega$} & \multirow{2}{*}{ Expectation } & \multirow{2}{*}{ Variance } \\
\hline & 0.1 & 0.2 & 0.3 & 0.4 & 0.5 & 0.6 & 0.7 & 0.8 & 0.9 & & \\
\hline 0.1 & 0.656798 & 0.761792 & 0.800713 & 0.823223 & 0.838534 & 0.84989 & 0.858785 & 0.866018 & 0.872063 & 0.814202 & 09035 \\
\hline 0.2 & 0.666862 & 0.767547 & 0.804938 & 0.826601 & 0.841356 & 0.852313 & 0.860904 & 0.867897 & 0.873745 & 0.818018 & 349224 \\
\hline 0.3 & 0.676707 & 0.773185 & 0.809085 & 0.829921 & 0.844133 & 0.854701 & 0.862994 & 0.869751 & 0.875407 & 0.821765 & 0.004011782 \\
\hline 0.4 & 0.686333 & 0.778706 & 0.813154 & 0.833184 & 0.846867 & 0.857053 & 0.865055 & 0.871581 & 0.877048 & 0.825442 & 0.003695726 \\
\hline 0.5 & 0.695741 & 0.784113 & 0.817147 & 0.836391 & 0.849557 & 0.85937 & 0.867088 & 0.873387 & 0.878669 & 0.829051 & 0.003400081 \\
\hline 0.6 & 0.704931 & 0.789406 & 0.821064 & 0.839543 & 0.852203 & 0.861652 & 0.869092 & 0.87517 & 0.88027 & 0.832592 & 0.003123881 \\
\hline 0.7 & 0.713903 & 0.794587 & 0.824906 & 0.842639 & 0.854808 & 0.863901 & 0.871069 & 0.876929 & 0.881852 & 0.836066 & 0.002866176 \\
\hline 0.8 & 0.722659 & 0.799657 & 0.828675 & 0.845681 & 0.85737 & 0.866116 & 0.873017 & 0.878666 & 0.883414 & 0.839473 & 0.002626033 \\
\hline 0.9 & 0.731199 & 0.804618 & 0.832371 & 0.84867 & 0.859891 & 0.868298 & 0.874939 & 0.880379 & 0.884956 & 0.842814 & 0.00240254 \\
\hline
\end{tabular}

TABle 9: Bank network under the index network-the sensitivity analysis of the influence of $\rho$ and $\omega$ on $\lambda_{B}^{*}$.

\begin{tabular}{cccccccccccc}
\hline$\rho$ & \multicolumn{10}{c}{$\omega$} & \multicolumn{1}{c}{$\omega$} \\
& 0.1 & 0.2 & 0.3 & 0.4 & 0.5 & 0.6 & 0.7 & 0.8 & 0.9 & Expectation & Variance \\
\hline 0.1 & $3.37 E-12$ & $3.75 E-08$ & $8.59 E-07$ & $4.91 E-06$ & $1.56 E-05$ & $3.64 E-05$ & $7.00 E-05$ & 0.000119 & 0.000184 & $4.78 E-05$ & $4.22 E-09$ \\
0.2 & $8.75 E-12$ & $6.02 E-08$ & $1.20 E-06$ & $6.35 E-06$ & $1.93 E-05$ & $4.35 E-05$ & $8.17 E-05$ & 0.000136 & 0.000207 & $5.50 E-05$ & $5.39 E-09$ \\
0.3 & $2.20 E-11$ & $9.54 E-08$ & $1.65 E-06$ & $8.17 E-06$ & $2.37 E-05$ & $5.19 E-05$ & $9.52 E-05$ & 0.000155 & 0.000234 & $6.33 E-05$ & $6.88 E-09$ \\
0.4 & $5.34 E-11$ & $1.49 E-07$ & $2.26 E-06$ & $1.05 E-05$ & $2.91 E-05$ & $6.17 E-05$ & 0.000111 & 0.000177 & 0.000263 & $7.27 E-05$ & $8.74 E-09$ \\
0.5 & $1.26 E-10$ & $2.30 E-07$ & $3.08 E-06$ & $1.33 E-05$ & $3.55 E-05$ & $7.31 E-05$ & 0.000128 & 0.000202 & 0.000295 & $8.34 E-05$ & $1.11 E-08$ \\
0.6 & $2.87 E-10$ & $3.52 E-07$ & $4.16 E-06$ & $1.69 E-05$ & $4.32 E-05$ & $8.63 E-05$ & 0.000148 & 0.00023 & 0.000331 & $9.55 E-05$ & $1.40 E-08$ \\
0.7 & $6.34 E-10$ & $5.30 E-07$ & $5.58 E-06$ & $2.12 E-05$ & $5.23 E-05$ & 0.000102 & 0.000171 & 0.00026 & 0.00037 & $1.09 E-04$ & $1.75 E-08$ \\
0.8 & $1.36 E-09$ & $7.91 E-07$ & $7.43 E-06$ & $2.66 E-05$ & $6.31 E-05$ & 0.000119 & 0.000197 & 0.000295 & 0.000414 & $1.25 E-04$ & $2.20 E-08$ \\
0.9 & $2.86 E-09$ & $1.17 E-06$ & $9.83 E-06$ & $3.32 E-05$ & $7.59 E-05$ & 0.00014 & 0.000226 & 0.000333 & 0.000462 & $1.42 E-04$ & $2.74 E-08$ \\
\hline
\end{tabular}

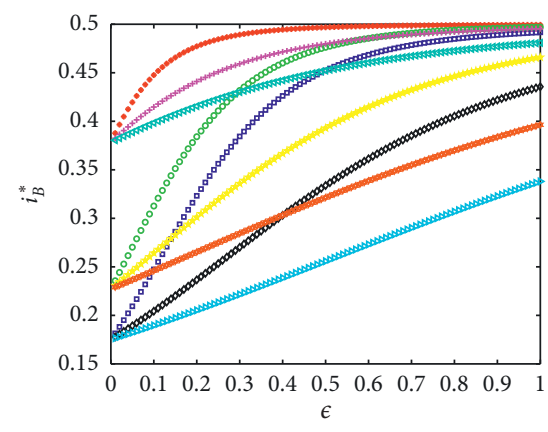

- The BA network, $\omega=0.2$

- The WS small-world network, $\omega=0.2$

- The index network, $\omega=0.2$

- The BA network, $\omega=0.5$

+ The WS small-world network, $\omega=0.5$

The index network, $\omega=0.5$

> The BA network, $\omega=0.7$

$\triangleleft$ The WS small-world network, $\omega=0.7$

* The index network, $\omega=0.7$

(a)

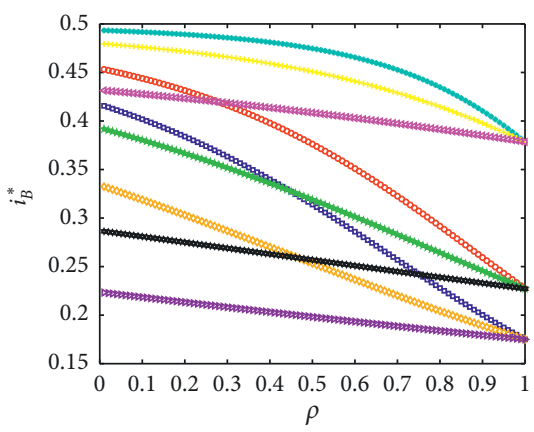

- The BA network, $\omega=0.3$

- The WS small-world network, $\omega=0.3$

- The index network, $\omega=0.3$

- The BA network, $\omega=0.5$

The WS small-world network, $\omega=0.5$

* The index network, $\omega=0.5$

D The BA network, $\omega=0.8$

$\checkmark$ The WS small-world network, $\omega=0.8$

* The index network, $\omega=0.8$

(b)

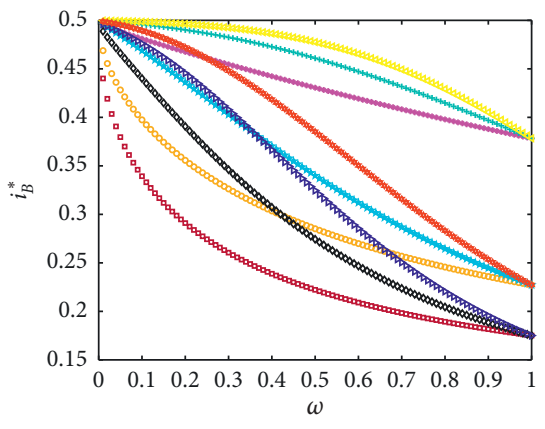

- The BA network, $\varepsilon=0.25$

- The WS small-world network, $\varepsilon=0.25$

The index network, $\varepsilon=0.25$

- The BA network, $\varepsilon=0.5$

* The WS small-world network, $\varepsilon=0.5$

* The index network, $\varepsilon=0.5$

D The BA network, $\varepsilon=0.75$

The WS small-world network, $\varepsilon=0.75$

* The index network, $\varepsilon=0.75$

(c)

FIgURe 7: Evolutionary laws of credit risk contagion scale $i_{B}^{*}$ of bank network counterparties. (a) The impact of bank counterparty business association degree $\varepsilon$ on $i_{B}^{*}$ in the bank network under different network structures and different bank counterparty risk resistance abilities $\omega$. (b) The impact of the bank's counterparty risk information processing ability $\rho$ on $i_{B}^{*}$ in the bank network under different network structures and different bank counterparty risk resistance abilities $\omega$. (c) The impact of bank counterparty risk resistance ability $\omega$ on $i_{B}^{*}$ in the bank network under different network structures and different bank counterparty business correlations $\varepsilon$.

addition, Figure $7(\mathrm{~b})$ shows that with the increase in the processing capacity $\rho$ of the bank counterparty risk information, the scale of the bank counterparty credit risk contagion $i_{B}^{*}$ continues to decrease. Figures $7(\mathrm{a})-7(\mathrm{c})$ depict that the scale of credit risk contagion $i_{B}^{*}$ of bank counterparties continues to decrease with the increase in the risk resistance capacity $\omega$ of bank counterparties. That is, the increase in the processing capacity $\rho$ of the bank 


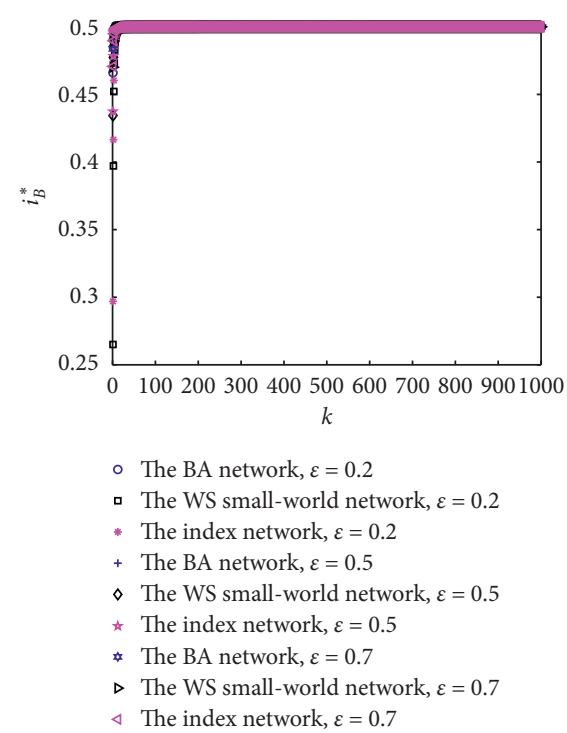

(a)

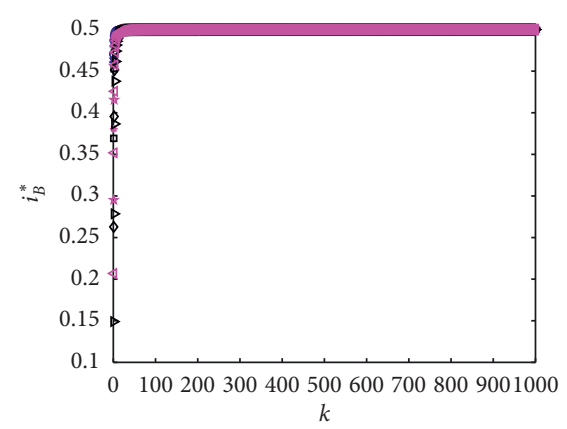

- The BA network, $\omega=0.3$

- The WS small-world network, $\omega=0.3$

* The index network, $\omega=0.3$

+ The BA network, $\omega=0.5$

$\diamond$ The WS small-world network, $\omega=0.5$

* The index network, $\omega=0.5$

* The BA network, $\omega=0.8$

$\triangle$ The WS small-world network, $\omega=0.8$

$\triangleleft$ The index network, $\omega=0.8$

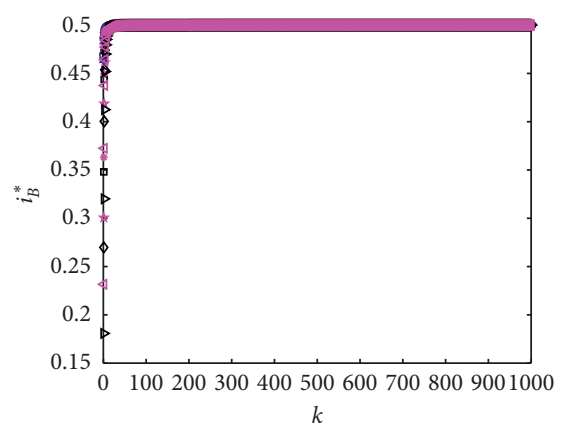

- The BA network, $\rho=0.25$

- The WS small-world network, $\rho=0.25$

* The index network, $\rho=0.25$

+ The BA network, $\rho=0.5$

$\diamond$ The WS small-world network, $\rho=0.5$

* The index network, $\rho=0.5$

* The BA network, $\rho=0.75$

$\triangleright$ The WS small-world network, $\rho=0.75$

$\triangleleft$ The index network, $\rho=0.75$

FiguRE 8: Evolutionary characteristics of the effect of the degree $k$ of the enterprise's counterparty on $i_{B}^{*}$. (a) The degree $k$ of the corporate counterparty on $i_{B}^{*}$ in the bank network under different network structures and different levels of bank transaction counterparty business association $\varepsilon$. (b) The degree $k$ of the corporate counterparty on $i_{B}^{*}$ in the bank network under different network structures and different bank counterparty risk resistance abilities $\omega$. (c) The degree $k$ of the corporate counterparty on $i_{B}^{*}$ in the bank network under different network structures and bank's counterparty risk information processing abilities $\rho$.

counterparty risk information and the bank counterparty risk resistance capacity $\omega$ will inhibit the spread of bank counterparty credit risk.

$\lambda_{A}=0.1, \lambda_{B}=0.001, \lambda_{B}=0.001,\left\langle k_{B}\right\rangle=9, \Theta_{1}^{*}=0.5$, $\lambda_{A \longrightarrow B}=0.09, m=5, k_{A}=k_{B}=1000, \mathrm{u}_{A}=\eta_{A}=\phi_{A}=0.2$, and $\eta_{B}=u_{B}=\phi_{B}=0.2$ were assumed to analyze the influence mechanism of the degree $k$ of the enterprise's creditrelated network on the credit risk spread $i_{B}^{*}$ of the bank's counterparty, considering that the bank network is fixed. Under the assumptions of the above parameters, the impact of the degree $k$ of the corporate credit-related network on $i_{B}^{*}$ under different network structures and different bank transaction counterparty business connection degrees $\varepsilon$ and under $\omega=\rho=0.3$ was initially explored. Second, the impact of the degree $k$ of the corporate credit-related network on $i_{B}^{*}$ under different network structures and different bank counterparty risk resistance capabilities $\omega$ and under $\varepsilon=\rho=0.4$ was investigated. Last, the impact and the degree $k$ of the enterprise's credit-related network on $i_{B}^{*}$ under different network structures and different processing capabilities of bank counterparty risk information $\rho$ and under $\varepsilon=\omega=0.5$ were examined.

Figure 8 shows that an increase in the degree $k$ of the enterprise's counterparty will cause an increase in the scale $i_{B}^{*}$ of the credit risk contagion of the bank's counterparty. Similarly, the bank credit association network is under three different network structures, with the increase in the degree $k$ of corporate counterparties, and the scale $i_{B}^{*}$ of credit risk transmission of bank counterparties will tend to a stable value.

$\lambda_{A}=0.1, \lambda_{B}=0.001,\left\langle k_{A}\right\rangle=9, \Theta_{1}^{*}=0.5, \lambda_{A \longrightarrow B}=0.09$, $m=5, k_{A}=k_{B}=1000, \mathrm{u}_{A}=\eta_{A}=\phi_{A}=0.2$, and $\eta_{B}=u_{B}=$ $\phi_{B}=0.2$ were assumed to analyze the influence mechanism of the degree $k$ of the bank network on the credit risk spread $i_{B}^{*}$ of the bank's counterparty, considering that the enterprise credit-related network is fixed. Under the assumption of the above parameters, the influence of the degree $k$ of bank network on $i_{B}^{*}$ under $\omega=\rho=0.3$ was initially explored under different network structures and different degrees of bank counterparty business association $\varepsilon$. Second, the influence of the degree $k$ of the bank network on $i_{B}^{*}$ under $\varepsilon=\rho=0.4$ was investigated under different network structures and different bank counterparty risk resistance capabilities $\omega$. Last, the influence of the degree $k$ of the bank network on $i_{B}^{*}$ under $\varepsilon=\omega=0.5$ was examined under different network structures and different processing capabilities of bank counterparty risk information $\rho$.

Figure 9 shows that when the enterprise's credit-related network is fixed, the credit risk contagion scale $i_{B}^{*}$ of the bank's counterparty increases continuously and the margin decreases with the increase in the degree $k$ of the bank's counterparty.

$\lambda_{B}=0.001,\left\langle k_{A}\right\rangle=9, \Theta_{1}^{*}=0.5, \lambda_{A \longrightarrow B}=0.09, m=5$, $k_{B}=1000, \quad u_{A}=\eta_{A}=\phi_{A}=0.2$, and $\eta_{B}=u_{B}=\phi_{B}=0.2$ were assumed to analyze the impact of the credit risk contagion rate $\lambda_{A}$ of the corporate credit-related network transaction counterparty, the risk contagion probability $\lambda_{A \longrightarrow B}$ of the corporate credit-related network CRT to the bank network on the scale $i_{B}^{*}$ of the bank counterparty credit risk contagion. The impact of the credit risk contagion rate $\lambda_{A}$ of the counterparties of corporate credit-related networks on $i_{B}^{*}$ in the bank network under different network structures was initially explored. Second, considering that $\lambda_{A}=0.1, \lambda_{B}=0.001,\left\langle k_{A}\right\rangle=9, \Theta_{1}^{*}=0.5, m=5, k_{B}=1000$, $u_{A}=\eta_{A}=\phi_{A}=0.2$, and $\eta_{B}=u_{B}=\phi_{B}=0.2$, the influence 


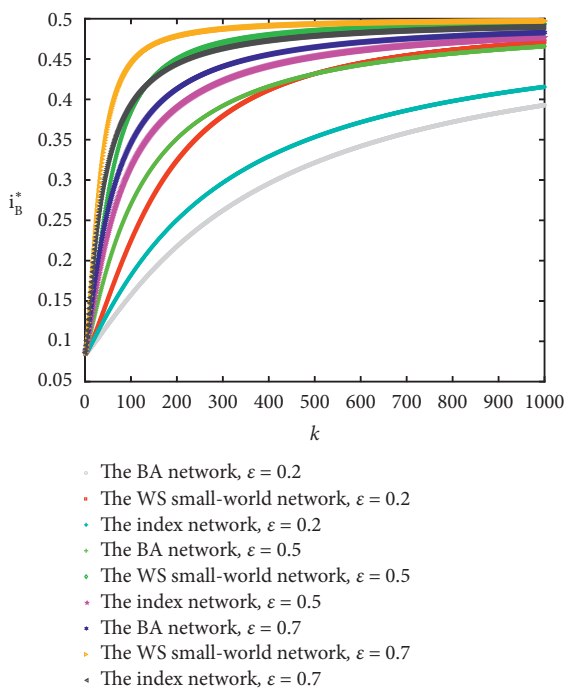

(a)

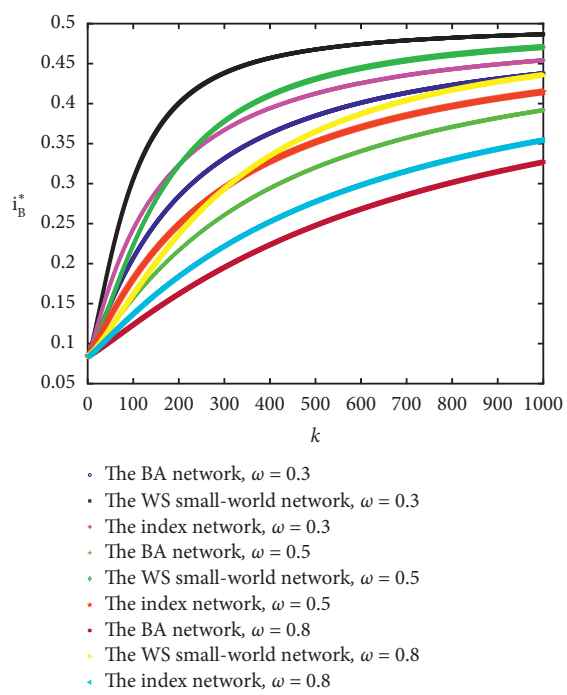

(b)

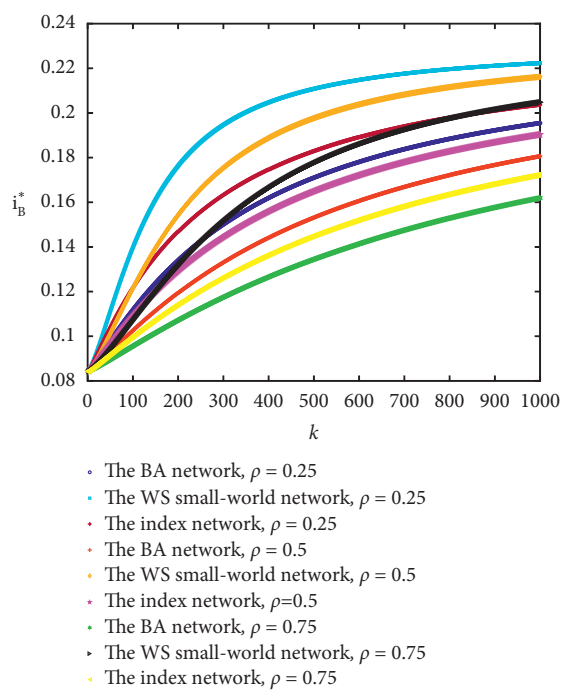

(c)

FIGURE 9: Evolutionary characteristics of the impact of bank transaction counterparty degree $k$ on $i_{B}^{*}$. (a) The impact of bank counterparty's degree $k$ on $i_{B}^{*}$ under different network structures and different bank transaction counterparty business connection degrees $\varepsilon$. (b) The impact of bank counterparty's degree $k$ on $i_{B}^{*}$ under different network structures and different bank counterparty risk resistance abilities $\omega$. (c) The impact of bank counterparty's degree $k$ on $i_{B}^{*}$ under different network structures and bank's counterparty risk information processing ability $\rho$.

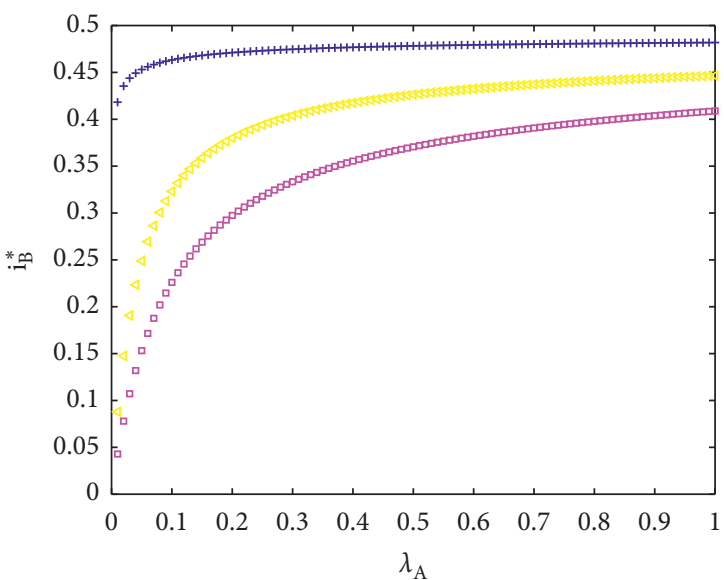

The index network

- The BA network

+ The WS small-world network

(a)

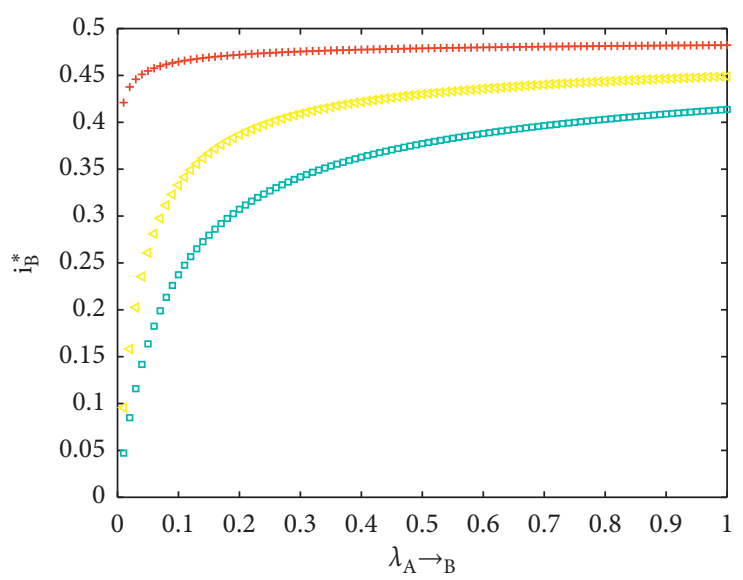

The index network

- The BA network

+ The WS small-world network

(b)

Figure 10: Evolutionary characteristics of bank counterparty credit risk contagion scale $i_{B}^{*}$. (a) The influence of the credit risk contagion rate $\lambda_{A}$ of the counterparty of the enterprise credit-related network on $i_{B}^{*}$ in the bank network under different network structures. (b) The influence of risk contagion probability $\lambda_{A \longrightarrow B}$ of the credit risk of corporate credit-related networks to the bank network on $i_{B}^{*}$ in the bank network under different network structures.

of the risk contagion probability $\lambda_{A \longrightarrow B}$ of the credit risk of corporate credit-related networks to the bank network on $i_{B}^{*}$ in the bank network under different network structures was examined.

Figures 10(a)-10(b) depict that the bank credit association network is under two kinds of network structures, namely, the influence mechanism and evolution characteristics of the credit risk contagion rate $\lambda_{A}$ of corporate credit associated network counterparties and the risk contagion probability $\lambda_{A \longrightarrow B}$ of corporate network credit risk contagion to bank network on bank counterparty credit risk contagion scale $I_{B}^{*}$. Figure 10 not only verifies Proposition 3 but also shows that the scale of credit risk contagion of bank counterparties in the WS network is greater than that of credit risk contagion of bank counterparties in the index network. In addition, the scale of credit risk contagion of bank counterparties in the 
index network is greater than that of bank transactions in the BA network. This result is because the heterogeneity of the BA network is greater than that of the index network, whereas the heterogeneity of the index network is greater than that of the WS network. If the heterogeneity of the banking network is strong, then bank counterparties less likely have credit risk and the scale of bank credit risk is small.

\section{Conclusion}

In the modern economic system, the business relationship between banks and nonbank enterprises based on credit association is increasingly close, and a significant symbiotic relationship is observed between banks and enterprises. However, the subject transactions based on credit become increasingly frequent with the in-depth development of the business relationship between banks and enterprises. Moreover, the credit relationship between banks and enterprises based on credit relationships is becoming increasingly complex and has become an important channel for credit risk contagion. Simultaneously, the credit risk contagion of bank and corporate counterparties is affected by many factors, which are the key and core of the risk management of the entire financial system. Therefore, this paper constructs a twolayer network model of credit risk contagion between the bank and corporate counterparties based on the perspective that banks do not withdraw loans from enterprises, considering the contagion effect of corporate credit default on their counterparties under credit association. In the case that banks do not withdraw loans from enterprises, the influence mechanism of the factors on the credit risk contagion evolution of bank-enterprise counterparties under the doublelayer network is analyzed through the theoretical analysis of factors (e.g., the coping capacity, risk appetite, influence, interenterprise credit risk contagion level and network heterogeneity in the interenterprise credit-related network, the risk prevention and control capabilities, business relevance, interbank the level of credit risk contagion and its network heterogeneity, as well as the level of credit risk contagion between bank-enterprise counterparty credit-related networks). In addition, the characteristics of the evolution of the credit risk contagion evolution of bank-enterprise counterparties under the double-layer network are examined. The main research conclusions are as follows:

(1) In the interenterprise credit association network, the threshold of credit risk contagion rate is positively correlated with the marginal increase in risk perception and risk leveling ability of the enterprise but negatively correlated with the marginal decrease in the initial economic impact, leverage level, and influence of the enterprise. Moreover, the contagion scale of the counterparty credit risk of an enterprise is negatively correlated with its risk perception level and risk leveling ability but positively correlated with its initial economic impact level, leverage level, and influence.

(2) In the interbank credit correlation network, the threshold of credit risk contagion rate is negatively correlated with the marginal decrease in the degree of interbank business correlation but positively correlated with the marginal increase in the bank's risk resistance and risk information processing capability. Moreover, the scale of credit risk contagion of bank counterparties is positively correlated with the degree of interbank business association but negatively correlated with the bank's ability to resist risks and the ability to process risk information.

(3) If the heterogeneity of the credit-related network of bank-enterprise counterparties is high, then the threshold of credit risk transmission rate is also high, and the scale of credit risk diffusion is low. Moreover, the scale of credit risk contagion of bank counterparties is positively correlated with the marginal decrease in the degree of corporate and bank counterparties.

(4) The credit risk contagion scale of a bank's counterparty is a monotonically increasing convex function of the credit risk contagion rate in corporate credit-related networks and the credit risk contagion rate among bank-enterprise networks.

The research conclusion of this study provides a theoretical basis and simulation model for the regulators to prevent the credit risk contagion of bank and business counterparties, which has high theoretical value and practical significance. However, in the real financial market, other influencing factors still affect the credit risk contagion of bank and corporate counterparties, which is also an important direction for our further research in the future.

\section{Data Availability}

The method in this article is computer mathematical simulation. Numerical simulation analysis is the most effective way to test real-time dynamic data without a large number of empirical validations. The authors simulate to explore the characteristics of the evolution of counterparty credit risk contagion of bank and corporate counterparties under the double-layer network by using Matlab2016b software. This paper does not have the data that can be obtained because they directly use the plot function of Matlab2016b software to make the images.

\section{Conflicts of Interest}

The authors declare that they have no conflicts of interest.

\section{Authors' Contributions}

Tingqiang Chen, Qinghao Yang, and Yutong Wang contributed equally to this work. They are co-first authors.

\section{Acknowledgments}

This work was supported by the National Natural Science Foundation of China (nos. 71871115, 71501094, and 71501131), the Major Project of Philosophy and Social 
Science Research in Colleges and Universities in Jiangsu Province (no. 2019SJZDA035), and the Outstanding Innovation Team of Philosophy and Social Science Research in Colleges and Universities of Jiangsu Province (no. 2017ZSTD005).

\section{References}

[1] T. Chen, J. He, and J. Wang, "Bifurcation and chaotic behavior of credit risk contagion based on Fitzhugh-nagumo system," International Journal of Bifurcation and Chaos, vol. 23, no. 7, Article ID 1350117, 2013.

[2] T. Lux, "A model of the topology of the bank-firm credit network and its role as channel of contagion," Journal of Economic Dynamics and Control, vol. 66, pp. 36-53, 2016.

[3] J. He, X. Sui, and S. Li, "An endogenous model of the credit network," Physica A: Statistical Mechanics and Its Applications, vol. 441, pp. 1-14, 2016.

[4] J. C. González-Avella, V. H. de Quadros, and J. R. Iglesias, "Network topology and interbank credit risk," Chaos, Solitons \& Fractals, vol. 88, pp. 235-243, 2016.

[5] A. Herman, D. Igan, and J. Solé, "The macroeconomic relevance of bank and nonbank credit: an exploration of U.S. data," Journal of Financial Stability, vol. 32, pp. 124-141, 2017.

[6] D. Ding, L. Han, and L. Yin, "Systemic risk and dynamics of contagion: a duplex inter-bank network," Quantitative Finance, vol. 17, no. 9, pp. 1435-1445, 2017.

[7] T. C. Silva, M. d. S. Alexandre, and B. M. Tabak, "Bank lending and systemic risk: a financial-real sector network approach with feedback," Journal of Financial Stability, vol. 38, pp. 98-118, 2018.

[8] T. Chen, J. Wang, H. Liu, and Y. He, "Contagion model on counterparty credit risk in the CRT market by considering the heterogeneity of counterparties and preferential-random mixing attachment," Physica A: Statistical Mechanics and Its Applications, vol. 520, pp. 458-480, 2019.

[9] G. D. Masi and M. Gallegati, "Bank-firms topology in Italy," Empirical Economics, vol. 43, no. 2, pp. 851-866, 2012.

[10] D. Asanuma, "Lending attitude as a financial accelerator in a credit network economy," Journal of Economic Interaction \& Coordination, vol. 8, no. 2, pp. 231-247, 2013.

[11] D. D. Gatti, M. Gallegati, B. Greenwald, A. Russo, and J. E. Stiglitz, "The financial accelerator in an evolving credit network," Journal of Economic Dynamics and Control, vol. 34, no. 9, pp. 1627-1650, 2010.

[12] L. Wang, S. Li, and T. Chen, "Investor behavior, information disclosure strategy and counterparty credit risk contagion," Chaos, Solitons \& Fractals, vol. 119, pp. 37-49, 2019.

[13] T. Chen, Y. Wang, Q. Zeng, and J. Luo, "Network model of credit risk contagion in the interbank market by considering bank runs and the fire sale of external assets," Physica A: Statistical Mechanics and Its Applications, vol. 542, pp. 1-17, 2020.

[14] X. Sui, L. Li, and X. Chen, "Risk contagion caused by interactions between credit and guarantee networks," Physica A: Statistical Mechanics and Its Applications, vol. 539, pp. 1-10, 2020.

[15] V. Fanelli and L. Maddalena, "A nonlinear dynamic model for credit risk contagion," Mathematics and Computers in Simulation, vol. 174, pp. 45-58, 2020.

[16] Z. Yin, M. Qiu, and L. Gan, "Information contents of collateral under heterogeneous borrower qualities on the bank loans market in China," China Economic Review, vol. 57, pp. 1-12, 2019.
[17] L.-W. Liang, B.-Y. Huang, C.-F. Liao, and Y.-T. Gao, "The impact of SMEs' lending and credit guarantee on bank efficiency in South Korea," Review of Development Finance, vol. 7, no. 2, pp. 134-141, 2017.

[18] R. Gropp, A. Guettler, and V. Saadi, "Public bank guarantees and allocative efficiency," Journal of Monetary Economics, pp. 1-17, 2019.

[19] F. Allen and E. Carletti, "Credit risk transfer and contagion," Journal of Monetary Economics, vol. 53, no. 1, pp. 89-111, 2006.

[20] A. Banal-Estañol, M. Ottaviani, and A. Winton, "The flip side of financial synergies: coinsurance versus risk contamination," Review of Financial Studies, vol. 26, no. 12, pp. 3142-3181, 2013.

[21] G. Gabbi, G. Iori, S. Jafarey, and J. Porter, "Financial regulations and bank credit to the real economy," Journal of Economic Dynamics and Control, vol. 50, pp. 117-143, 2015.

[22] T. Chen, X. Li, and J. Wang, "Spatial interaction model of credit risk contagion in the CRT market," Computational Economics, vol. 46, no. 4, pp. 519-537, 2015.

[23] G. Cimini and M. Serri, "Entangling credit and funding shocks in interbank markets," PLoS One, vol. 11, no. 8, Article ID e0161642, 2016.

[24] L. Wang, S. Li, and C. Wu, "Risk contagion in inter-firm credit guarantee network," Physica A: Statistical Mechanics and Its Applications, vol. 526, pp. 1-12, 2019.

[25] S. Li and X. Sui, "Contagion risk in endogenous financial networks," Chaos, Solitons \& Fractals, vol. 91, pp. 591-597, 2016.

[26] S. Mohammad, M. Asutay, R. Dixon, and E. Platonova, "Liquidity risk exposure and its determinants in the banking sector: a comparative analysis between Islamic, conventional and hybrid banks," Journal of International Financial Markets, Institutions and Money, vol. 66, pp. 1-20, 2020.

[27] D. J. Watts and S. H. Strogatz, "Collective dynamics of "smallworld” networks," Nature, vol. 393, no. 6684, pp. 440-442, 1998.

[28] A.-L. Barabási and R. Albert, "Emergence of scaling in random networks," Science, vol. 286, no. 5439, pp. 509-512, 1999.

[29] M. E. J. Newman, C. Moore, and D. J. Watts, "Mean-field solution of the small-world network model," Physical Review Letters, vol. 84, no. 14, pp. 3201-3204, 2000.

[30] V. Y. Guleva, M. V. Skvorcova, and A. V. Boukhanovsky, "Using multiplex networks for banking systems dynamics modelling," Procedia Computer Science, vol. 66, pp. 257-266, 2015.

[31] T. Chen, B. Xiao, and H. Liu, "Credit risk contagion in an evolving network model integrating spillover effects and behavioral interventions," Complexity, vol. 2018, Article ID 1843792, 17 pages, 2018.

[32] J. I. Halvorsen and D. H. Jacobsen, "How important can bank lending shocks be for economic fluctuations?" The North American Journal of Economics and Finance, vol. 29, pp. 104-123, 2014.

[33] T. C. Silva, M. A. da Silva, and B. M. Tabak, "Systemic risk in financial systems: a feedback approach," Journal of Economic Behavior \& Organization, vol. 144, pp. 97-120, 2017.

[34] Q. Qian, Y. Yang, J. Gu, and H. Feng, "Information authenticity, spreading willingness and credit risk contagion-a dual-layer network perspective," Physica A: Statistical Mechanics and Its Applications, vol. 536, pp. 1-13, 2019.

[35] X. Chen, W. Li, S. Hu, and X. Liu, "Quality of information disclosure, property rights, and bank loans: a bank heterogeneity perspective," China Journal of Accounting Research, vol. 12, no. 1, pp. 63-92, 2019. 
[36] L. Bargigli and M. Gallegati, "Random digraphs with given expected degree sequences: a model for economic networks," Journal of Economic Behavior \& Organization, vol. 78, no. 3, pp. 396-411, 2011.

[37] M. Montagna and C. Kok, Multi-Layered Interbank Model for Assessing Systemic Risk. Kiel Working Papers, Kiel Institute for the World Economy, Kiel, Germany, 2013.

[38] S. Jiang and H. Fan, "Credit risk contagion coupling with sentiment contagion," Physica A: Statistical Mechanics and Its Applications, vol. 512, pp. 186-202, 2018.

[39] Y. Li and Z. Zhou, "Associated credit risk contagion between enterprises based on the imperfect immunization scenario," Procedia Computer Science, vol. 55, pp. 336-340, 2015.

[40] A. Krause and S. Giansante, "Interbank lending and the spread of bank failures: a network model of systemic risk," Journal of Economic Behavior \& Organization, vol. 83, no. 3, pp. 583-608, 2012.

[41] C. Memmel and A. Sachs, "Contagion in the interbank market and its determinants," Journal of Financial Stability, vol. 9, no. 1, pp. 46-54, 2012.

[42] R. Burkholz, M. V. Leduc, A. Garas, and F. Schweitzer, "Systemic risk in multiplex networks with asymmetric coupling and threshold feedback," Physica D: Nonlinear Phenomena, vol. 323-324, pp. 64-72, 2016.

[43] L. Wang, S. Li, J. Wang, and Y. Meng, "Real estate bubbles in a bank-real estate loan network model integrating economic cycle and macro-prudential stress testing," Physica A: Statistical Mechanics and Its Applications, vol. 542, pp. 1-16, 2020.

[44] K. Anand, P. Gai, S. Kapadia, S. Brennan, and M. Willison, “A network model of financial system resilience," Journal of Economic Behavior \& Organization, vol. 85, pp. 219-235, 2013.

[45] C. Granell, S. Gómez, and A. Arenas, "Dynamical interplay between awareness and epidemic spreading in multiplex networks," Physical Review Letters, vol. 111, no. 12, pp. 1-10, 2013.

[46] G. Iori, R. N. Mantegna, L. Marotta, S. Miccichè, J. Porter, and M. Tumminello, "Networked relationships in the e-MID interbank market: a trading model with memory," Journal of Economic Dynamics and Control, vol. 50, pp. 98-116, 2015.

[47] A.-C. Hüser, G. Hałaj, C. Kok, C. Perales, and A. van der Kraaij, "The systemic implications of bail-in: a multi-layered network approach," Journal of Financial Stability, vol. 38, pp. 81-97, 2018.

[48] Y. Dong, J. Wang, and T. Chen, "Price linkage rumors in the stock market and investor risk contagion on bilayer-coupled networks," Complexity, vol. 2019, Article ID 4727868, 21 pages, 2019.

[49] G. Ferri and M. Messori, "Bank-firm relationships and allocative efficiency in Northeastern and Central Italy and in the South," Journal of Banking \& Finance, vol. 24, no. 6, pp. 1067-1095, 2000.

[50] N. Dwenger, F. M. Fossen, and M. Simmler, "Firms' financial and real responses to credit supply shocks: evidence from firm-bank relationships in Germany," Journal of Financial Intermediation, vol. 41, pp. 1-14, 2020.

[51] D. Barro and A. Basso, "Credit contagion in a network of firms with spatial interaction," European Journal of Operational Research, vol. 205, no. 2, pp. 459-468, 2010.

[52] Y. Luo, J. Wang, Y. Zhao, and T. Chen, "Scare behavior diffusion model of health food safety based on complex network," Complexity, vol. 2018, Article ID 5902105, 14 pages, 2018.
[53] A. V. Leonidov and E. L. Rumyantsev, "Default contagion risks in Russian interbank market," Physica A: Statistical Mechanics and Its Applications, vol. 451, pp. 36-48, 2016.

[54] I. Aldasoro, D. Delli Gatti, and E. Faia, "Bank networks: contagion, systemic risk and prudential policy," Journal of Economic Behavior \& Organization, vol. 142, pp. 164-188, 2017.

[55] H. Fout, G. Li, M. Palim, and Y. Pan, "Credit risk of low income mortgages," Regional Science and Urban Economics, vol. 80, pp. 1-17, 2020.

[56] L. Bargigli, G. di Iasio, L. Infante, F. Lillo, and F. Pierobon, "The multiplex structure of interbank networks," Quantitative Finance, vol. 15, no. 4, pp. 673-691, 2015.

[57] R. Gençay, H. Pang, M. C. Tseng, and Y. Xue, "Contagion in a network of heterogeneous banks," Journal of Banking \& Finance, vol. 111, pp. 1-18, 2020.

[58] A. Eross, A. Urquhart, and S. Wolfe, "Liquidity risk contagion in the interbank market," Journal of International Financial Markets, Institutions and Money, vol. 45, pp. 142-155, 2016.

[59] M. Bottero, S. Lenzu, and F. Mezzanotti, "Sovereign debt exposure and the bank lending channel: impact on credit supply and the real economy," Journal of International Economics, vol. 126, pp. 1-26, 2020.

[60] M. O. Jackson, "The Economics of Social Networks," Cambridge University Press, Cambridge, UK, 2006. 